\title{
Johannes de Sacrobosco und die Sphaera-Tradition in der katholischen Zensur der Frühen Neuzeit
}

\author{
Christoph Sander
}

Johannes des Sacrobosco and the Sphere Tradition in Early Modern Catholic Censorship

\begin{abstract}
Johannes de Sacrobosco's (c. 1195-c. 1256) On the Sphere, a short introduction into qualitative cosmology written in the thirteenth century, was the most widely used textbook on cosmology in the early-modern period, being reprinted, re-edited or commented over 320 times. While the reception and circulation of this work in the sixteenth and seventeenth century is well known, one fact has so far escaped the notice of scholars: Sphaera textbooks were subject to several acts of ecclesiastical censorship in the early modern period, even though the content of this work promoted a cosmology that opposed the allegedly heretical implications of Copernicanism. This paper investigates for the very first time the dynamics and motives behind Roman and Iberian censorship in relation to this cosmology treatise. Editions and commentaries published by Protestants were generally regarded as suspect, but rarely prohibited across-the-board. Instead, they were usually approved for scientific use after expurgations had removed problematic theological passages. However, the commentary (1550) authored by the Catholic Mauro da Firenze (1493-1556) was prohibited repeatedly and completely because it contained theologically dangerous ideas. The case studies presented in this paper shall shed light on the dynamics of knowledge within the Sphere tradition from a new perspective, that of the Catholic censorship of books. Moreover, a longitudinal study based on a specific genre of books provides insight into the ideology and practices of early modern catholic book censorship, whereby the well-known problematic relationship between science and religion in the pre-modern period is seen in the context of a confessionalisation of science.
\end{abstract}

Keywords: Sacrobosco, Censorship, Index of Prohibited Books, Astronomy, Cosmology

Johannes de Sacroboscos (c. 1195-c. 1256) De sphaera, eine Einführung in die Kosmologie aus dem 13. Jahrhundert, war mit über 320 Drucken das am häufigsten edierte, kommentierte oder adaptierte astronomischkosmologische Handbuch der Frühen Neuzeit. Während die Rezeption und Verbreitung dieses Werkes im 16. und 17. Jahrhundert bereits vielfach untersucht wurden ist bisher übersehen worden, dass diese vermeintlich unproblematischen Sphaera-Textbücher auch vielfach Gegenstand der katholischen Zensur wurden, obwohl sie gerade eine Kosmologie enthielten, die Katholiken als Bollwerk gegen den aufkommenden Kopernikanismus betrachteten. Vorliegender Aufsatz untersucht erstmals die Dynamik und Motive der zahlreichen präventiven und posthumen Zensuren der römischen und iberischen Index- und Zensurorgane mit Bezug auf diesen Kosmologietraktat. Von protestantischen Autoren verfasste Kommentare zur Sphaera waren zwar prinzipiell verdächtig, wurden aber selten pauschal verboten. Vielmehr wurden sie aufgrund ihres wissenschaftlichen Wertes meist genehmigt, nachdem geringfügige, theologiebezogene Expurgationen durchgeführt worden waren. Der Kommentar (1550) des katholischen Autors Mauro da Firenze (1493-1556) wurde hingegen wiederholt und prinzipiell verboten, weil er theologisch bedenkliche Gedanken enthielt. Die hier präsentierte Fallstudie soll zum einen die Wissensdynamik der frühneuzeitlichen Sphaera-Tradition aus einem neuen Blickwinkel beleuchten, und zwar dem der Buchzensur. Zum anderen gewährt ein buchbezogener Längsschnitt Einblicke in die Ideologie und Praktiken der katholischen Buchzensur, wodurch auch die bekanntermaßen problematische Beziehung von Wissen und Religion in der vormodernen Zeit im Lichte einer Konfessionalisierung der Wissenschaften erscheint.

Schlüsselwörter: Sacrobosco, Zensur, Index der Verbotenen Bücher, Astronomie, Kosmologie 
David Wootton hat kürzlich in seiner umfassenden wissenschaftshistorischen Studie The Invention of Science (2015) die Ansicht vertreten, dass der Einfluss der Religion auf die Akteure der sogenannten Wissenschaftlichen Revolution gemeinhin überbewertet wird (Wootton 2015: 575-577). ${ }^{1} \mathrm{Er}$ wendet sich hiermit gegen die These, dass beispielsweise die Annahme eines Schöpfergottes oder die mit dem Protestantismus einhergehenden Umwälzungen die Entstehung oder Festigung bestimmter wissenschaftlicher Konzepte oder Methoden begünstigt habe. Wootton stellt recht lapidar fest: „If Protestantism was what counted, [the Catholic] Galileo would not have been a great scientist." (Wootton 2015: 576) In der Tat positioniert sich der Historiker hier gegen eine historiographische Tradition, die den Einfluss der Religion auf die Dynamiken der Wissensorganisation und -produktion unterstreicht. So schreibt etwa Christoph Lüthy geradezu antithetisch und selbstbewusst über die "confessionalization of physics“:

When writing about the so-called „Scientific Revolution“, historians are inclined to describe the detachment of the observational fact from the old philosophico-theological edifice of natural philosophy as a victory of science, and notably of a mathematical and empirical mentality, over metaphysics and as a sign of practical progress in quantification, instrumentation, observation, and experimentation. But it could also be argued, on a more sombre note, that the new emphasis on facticity is just as much an act of scientific liberation as it is the effect of censorship from without and within, in the sense that it constituted the only safe way of speaking about the created world without hurting religious feelings and triggering unpleasant private or public reactions (Lüthy 2005: 108).

In dieser Perspektive erscheinen wesentliche, aus heutiger Sicht meist als „Errungenschaften“ bewertete wissenschaftliche Entwicklungen des 16. und 17. Jahrhunderts zumindest teilweise als Reflexe auf religiöse Spannungen in einer Zeit des konfessionellen Konflikts.

Gerade der Zensur, wie sie von katholischer Seite als Reaktion auf jenen konfessionellen Konflikt im 16. Jahrhundert mit der spanischen und römischen Inquisition institutionalisiert wurde, haftet immer noch der Geruch einer Organisation an, die die Entstehung neuen Wissens hemmte und fortschrittsfeindlich wirkte. Erst seit relativ kurzer Zeit ist durch die Edition der Zensurdokumente aus den römischen Archiven (2009, ediert in den bisher vier Bänden von Catholic Church and Modern Science) Wissenschafts- und Philosophiehistorikern die Möglichkeit verschafft worden, nicht nur einzelne und bekannte Fälle der vermeintlichen Wissenschaftszensur - wie etwa die von Giordano Bruno oder Galileo Galilei - zu extrapolieren, sondern in der Breite der Dokumente zu stöbern und so ein ausgewogeneres Bild 
davon zu zeichnen, welcher Einfluss dieser Form der Zensur überhaupt nachgesagt werden kann. ${ }^{2}$ Diese Aufarbeitung entscheidet nicht darüber, ob Wootton oder Lüthy Recht zu geben ist. Diese Aufarbeitung trägt vielmehr dazu bei, besser zu begreifen, wie die Zensoren tatsächlich vorgingen und welche Bereiche der Wissenschaftsproduktion der Frühen Neuzeit sie überhaupt ins Visier nahmen. Hierbei ist die Arbeit der Zensoren der Arbeit der Forscher nicht symmetrisch entgegengesetzt, es geht also nicht in erster Linie um Täter und Opfer der Zensur. Vielmehr ist die Zensur selbst in die Historiographie der Wissens- und Wissenschaftsgeschichte zu integrieren, als ein Dialog und Disput zwischen Klerikern im Auftrag der Zensur und der wissenschaftlichen Literaturproduktion, die auf Vorgaben reagierte oder diese präventiv umsetzte. Die Praktiken der Zensoren sind demnach als „Zensur“, „Begutachtung“, „Kritik“ und „review“ gleichermaßen zu betrachten (Biagioli 2002).

Hauptanliegen dieses Aufsatzes ist der Versuch, sich sich der Produktion astronomischen Wissens der Frühen Neuzeit aus dieser Perspektive der Zensur zu nähern. Die Verurteilung der astronomischen Lehre des Kopernikanismus, die ihren vorläufigen Höhepunkt 1633 im Prozess gegen Galileo Galilei (1564-1642) fand, ist vermutlich das berühmteste Beispiel des augenscheinlichen Konfliktes zwischen Wissenschaft und Kirche, zwischen Astronomie und Theologie in der Epoche der Frühen Neuzeit - ein Konflikt, der vielfach sogar als Paradigma der sogenannten Wissenschaftlichen Revolution betrachtet wurde (Finocchiaro 2005; Pagano 2009; Mayer 2015). Nikolaus Kopernikus' (1473-1543) Werk De revolutionibus (1543) wurde 1616 auf den Index der Verbotenen Bücher (Index librorum prohibitorum) gesetzt und einige angemahnte Korrekturen oder Schwärzungen, sogenannte Expurgationen, wurden 1620 präzise vorgeschrieben (Wolters 2015). Die jüngere Forschung hat das vermeintlich Revolutionäre an Kopernikus' Werk und seinen Ideen längst zu Gunsten eines umfassenderen Bildes der Kopernikusrezeption korrigiert (Omodeo 2014; Neuber et al. 2015). Aus druckgeschichtlicher Perspektive zeigt sich De revolutionibus ohnehin kaum als auflagenstarke Publikation, wenngleich das Werk von zahlreichen wichtigen Akteuren der Epoche minutiös studiert wurde (Gingerich 2004).

Bereits Arthur Koestler (1959) hat mit Blick auf De revolutionibus spöttisch von einem „worst seller" gesprochen und darauf hingewiesen, dass die Bedeutung von Kopernikus' Buch mit dem Klassikerwerk der ptolemäischen, geozentrischen Kosmologie verglichen werden müsse (Koestler 1959: 191): Johannes de Sacroboscos De sphaera, einer knappen Einführung in die qualitative Astronomie aus dem 13. Jahrhundert, das in zahlreichen Editionen seit 1472 wieder und wieder verlegt wurde. Auch Elizabeth L. Eisenstein (1979) hat herausgestellt, dass Buchdrucker der Frühen Neu- 
zeit vor allem dazu beigetragen haben, die ptolemäische Kosmologie zu popularisieren und bezieht sich hierbei ebenfalls auf die Verbreitung von Sacroboscos Werk (Eisenstein 2005: 615-616). ${ }^{3}$

Die überwältigende bibliometrische Evidenz des Druckerfolgs sogenannter Sphaera-Traktate im 15., 16. und 17. Jahrhundert ist in jüngerer Forschung vielfach katalogisiert, erörtert und untersucht worden (Pantin 1987; Ludwig 2010; Hamel 2004; Hamel 2014; Gingerich 1988; Gingerich 2015; Valleriani 2017; Sacrobosco 2008). Jüngste Erhebungen identifizieren nicht weniger als 320 Drucke aus jener geozentrischen Sacrobosco-Tradition, die seit der editio princeps (1472) nicht nur zahlreiche Nachdrucke in vielen Zentren Europas verzeichnet, sondern dutzende Kommentare zu Sacroboscos Traktat, umfassende Sammelausgaben, Übersetzungen in alle wichtigen westeuropäischen Volkssprachen sowie eigenständige, von Sacroboscos Text losgelöste Sphaera-Traktate (https://sphaera.mpiwgberlin.mpg.de). Von einer Tradition lässt sich hier nicht nur sprechen, weil alle Drucke an dasselbe mittelalterliche Werk anknüpfen. Vielmehr ist auch in der bisherigen Forschung bereits herausgestellt worden, dass viele der frühneuzeitlichen Sphaera-Editionen nicht unabhängig voneinander entstanden, sondern die Verleger und Buchdrucker untereinander kooperierten oder mit neuen Sphaera-Ausgaben an bestehende anknüpften, diese ergänzten, erweiterten und individuellen Anforderungen anpassten.

Obschon die astronomische Grundlage der Sphaera-Traktate im Wesentlichen ptolemäisch und die naturphilosophische Grundlage meist aristotelisch bleibt, handelt es sich bei diesem Text dennoch keineswegs um einen konservativen oder statischen Wissensspeicher (Pedersen 1985). Schon die mittelalterliche Vorlage berücksichtigt bereits jüngere arabische Werke der Astronomie und ist in den folgenden Jahrhunderten weiter ergänzt, aktualisiert und kritisch diskutiert worden. Der Name „Sacrobosco" wurde somit zunehmend zum Label für einen bestimmten Typus astronomischer und kosmologischer Schriften, die Wissen aus zahlreichen Forschungskontexten und Disziplinen bündelten, von Geographie und Nautik über Landwirtschaft und Astrologie bis hin zu Medizin (Oosterhoff 2015; Crowther et al. 2015; Valleriani 2017). Die Akteure dieser Dynamik stammten zudem aus unterschiedlichen geographischen/nationalen, akademischen und konfessionellen Kontexten. Kurzum, die Sphaera-Tradition bildet nicht nur ein Kooperationsnetzwerk von Verlegern ab, sondern umfasst, bezogen auf die Inhalte der Drucke, auch ein „Wissenssystem“ der Frühen Neuzeit.

Dass auch dieses Wissenssystem von der katholischen Zensur betroffen war, ist in der bisherigen Forschung kaum berücksichtigt worden. ${ }^{4}$ Weit vor Kopernikus' Werk (1543 gedruckt, 1616 verboten, 1620 expurgiert) untersuchten und mithin verboten katholische Zensurorgane Schriften aus 
der Sphaera-Tradition bereits in der zweiten Hälfte des 16. Jahrhunderts, dabei verstärkt ab den 1570er Jahren. Diese Zensurmaßnahmen zielten hingegen, anders als im Fall des Kopernikanismus, nicht auf spezifische astronomische oder kosmologische Lehren, sondern betrafen eher kontextuelle beziehungsweise akzidentielle Faktoren: etwa die Konfession des Autors oder dessen theologische Thesen, nicht aber kosmologische Weltvorstellungen als solche. Auch die Motivationen der Zensurmaßnahmen bleiben im Falle der Sphaera-Tradition oftmals im Dunkeln. Es handelt sich bei den hier untersuchten Fällen nicht um dramatische Prozesse der Inquisition, sondern eher um Produkte des in den Abläufen bürokratisch festgelegten business as usual der Zensurbehörden. ${ }^{5}$

Der in diesem Aufsatz angestrebte Querschnitt durch die Geschichte der katholischen Zensur zwischen 1550 und 1650 entlang der SphaeraTradition ist geleitet von zwei Erkenntniszielen: Zum einen (1) dient die Zuspitzung des Corpus auf die Sphaera-Tradition dazu, die katholische Zensur besser zu begreifen. Es soll also die Rationalität und Praxis der katholischen Index- und Zensurkongregation an einem konkreten Beispiel illustriert werden und somit zu einem besseren Verständnis beigetragen. Hier ergibt sich nämlich, dass die Leitlinien der katholischen Zensur sich nicht paradigmatisch durch ihre Auseinandersetzung mit dem Kopernikanismus verstehen lassen, sondern eher ideologische oder konfessionspolitische Zensurkriterien angelegt wurden. Gleichzeitig bezeugen die untersuchten Dokumente auch eine gewisse Toleranz der Zensurbehörden, die oftmals übersehen wird, wenngleich diese Toleranz in der Forschung längst bekannt ist und in vielen Studien untersucht worden ist (Reusch 1886; Wolf 2001; Green 2005; Caravale 2011; Fragnito 2001; Grendler 2007; Esteve 2018).

Zum anderen (2) bietet die Fallstudie die Möglichkeit, durch den Blick der Zensur auch die Sphaera-Tradition besser zu verstehen. Gerade weil jene Sphaera-Tradition in der kosmologischen Wissenskonfiguration der Frühen Neuzeit eine beträchtliche Rolle spielte, stellt sich etwa die Frage, ob die Zensurbehörden auf diese Wissensdynamiken nolens volens Einfluss nahmen und damit auch die Verbreitung kosmologischer Ideen steuerten und lenkten. Ugo Baldini hat beispielsweise herausgestellt, wie die katholische Zensur die Ablehnung der Astrologie als Wissenschaft mit begünstigte, ohne dass ihr hierbei eine klare Agenda oder explizite Zielsetzung zu unterstellen wäre (Baldini 2001). Auch die lokale und interkonfessionelle Verbreitung und Rezeption der Sphaera-Drucke, das heißt wesentliche Dynamiken innerhalb der Sphaera-Tradition, lassen sich durch den Längschnitt durch die Zensurgeschichte in den Blick nehmen, insofern auch die Ausübung einer Zensur sowie deren Be- oder Missachtung eine Form der Rezeption darstellt. 


\section{Begriffsklärung „Zensur”}

Während die kirchliche Zensur schriftlicher Produktion, akademischer Lehre und Predigt bereits im Mittelalter institutionalisierte Formen besaß, und auch die Buchzensur bereits zu Beginn des 16. Jahrhunderts päpstliche Legitimation erhielt, stellten doch die Gründung der römischen Glaubenskongregation 1542 sowie der Tridentinische Index der Verbotenen Bücher von 1564 entscheidende Wegmarken der katholischen Zensurgeschichte dar. ${ }^{6}$ Die römische Inquisition und Indexkongregation sowie die schon zuvor gegründete spanische (1478) beziehungsweise portugiesische (1515) Inquisition, die übergreifend als Organe der katholischen Zensur bezeichnet werden können, nahmen in bisher unbekanntem Ausmaß Einfluss auf Theologie, Wissenschaft, Kunst, Literatur und Gesellschaft der Frühen Neuzeit. Weil diese Institutionen bereits umfassend erforscht worden sind, werden rudimentäre Kenntnisse der Geschichte der römischen und iberischen Inquisition hier vorausgesetzt.

Es sollen dennoch zunächst einige wichtige begriffliche Unterscheidungen und Aspekte der Zensurpraxis und -theorie zusammengefasst werden, die für die folgende, die Sphaera-Tradition betreffende Zensurgeschichte unmittelbar relevant sind. Eine umfassende Definition des historischen Zensurbegriffs ist schwierig und strittig. Zensur lässt sich, heute wie damals und ganz abstrakt, einerseits als ein Herrschaftsinstrument beschreiben, das heißt, als ein Mittel, eine gewünschte Vormachtstellung zu garantieren, zu erhalten oder widerstreitende Theorien beziehungsweise Akteure zu unterminieren. Andererseits ist Zensur auch ein Instrument der Selbstkontrolle, das der inneren Geschlossenheit und der Korrektur von Irrtümern in einem Lehrgebäude dient.

Die katholische Zensur der Frühen Neuzeit umfasste Aspekte von beiden, mitnichten widersprüchlichen Ansprüchen. Sie richtete sich hierbei insbesondere gegen mannigfaltige Formen der religiösen Heterodoxie und Häresie, an erster Stelle sicherlich gegen den aufkommenden Protestantismus, aber auch gegen innerkatholische Reformbewegungen oder Gedankengut, das im Widerspruch zur päpstlichen Autorität oder der traditionellen Kirchenlehre betrachtet wurde (Neveu 1993; Arnold 2008). Die für die folgende Darstellung maßgebliche Form der Zensur war primär theologisch und konfessionspolitisch ausgerichtet, entsprechend zielte sie auf heterodoxe theologische Lehren beziehungsweise auf deren ketzerische Verbreiter. $^{7}$

Wenngleich die römische, spanische und portugiesische Inquisition als zentralistische Apparate auftraten und in ihrer Historiographie auch oft so beschrieben worden sind, handelte es sich jedoch nicht um monolithische, widerspruchsfreie, geschweige denn ahistorische Gebilde. Vielmehr 
unterlag die Praxis der Zensur ähnlichen Varietäten und Kontingenzen wie andere Apparate, die eine genuine Entwicklungsgeschichte haben, in denen zwischen Theorie und Praxis zu unterscheiden ist, die von internen Macht- und Einflussstreitereien geprägt waren und bei denen eine lokal unterschiedliche Anwendung bestehender Regeln beachtet werden muss (Godman 2000). ${ }^{8}$ Die bloße Tatsache der Koexistenz einer spanischen, portugiesischen und römischen Inquisition ist hierbei ein aussagekräftiges Indiz für die politische und konfessionelle Komplexität der Frühen Neuzeit. Alle diese drei Zensurbehörden wurden päpstlich eingerichtet oder genehmigt, und doch unterschieden sie sich mitunter in ihrer Zielsetzung, vor allem aber in ihren Methoden, ihrer internen Organisation und ihrer Jurisdiktion.

Die meisten textbezogenen Zensurmaßnahmen, so auch die katholischer Institutionen, lassen sich in posthume und präventive Maßnahmen unterteilen. $\mathrm{Zu}$ posthumer Zensur zählen im engeren Sinne das vollständige Verbot bestimmter oder sämtlicher Schriften eines Autors beziehungsweise die Streichung einzelner Abschnitte in einer Schrift. Während also die Werke eines Autors schlicht aufgrund seiner Biographie, Herkunft oder konfessioneller Zugehörigkeit verboten werden konnten, ja gar deren Vernichtung angemahnt wurde, zielten sogenannte Expurgationen nur auf problematische Textstellen innerhalb einer ansonsten freigegebenen Schrift. Posthume Zensuren sind nicht vom Autor selbst veranlasst, sondern vom jeweiligen Zensurorgan. Sie betrafen in der Frühen Neuzeit in der Regel gedruckte Bücher. Das einflussreichstes Instrument der katholischen Zensur war der immer wieder erweiterte und neu überarbeitet publizierte Index Librorum Prohibitorum (Index der Verbotenen Bücher), der jedoch unterschiedlicher regionaler Jurisdiktion unterlag, also Einfluss auf Buchmarkt, Buchhandel und Literaturproduktion bestimmter Gebiete nahm. Allerdings konnten individuelle oder universelle Sondergenehmigungen vom jeweiligen Zensurorgan erteilt werden, auch auf dem Index platzierte oder anderweitig verdächtige Schriften lesen zu dürfen (sogenannte licentiae). Posthume Zensuren lassen sich nicht nur von den publizierten Indices ausgehend nachverfolgen, sondern auch über interne handschriftliche Dokumente, die Genese und mithin abweichende lokale Anwendungen bezeugen. Zudem dokumentieren erhaltene, nachträglich expurgierte Buchexemplare aufschlussreiche Spuren, die über die Praxis der Zensur informieren (Schmuki 1999; Oosterhoff 2015).

Im Gegensatz hierzu spricht man von präventiver Zensur (censura praevia) in Fällen, in denen eine Schrift vor ihrem Druck einer Begutachtung und gegebenenfalls einer Korrektur unterzogen wurde. Diese Form der Zensur wurde meist vom Autor, dem Verleger oder der religiösen Körperschaft, etwa des Ordens des Autors, veranlasst. In zahlreichen gedruckten 
Werken der Frühen Neuzeit findet sich somit, meist zu Beginn oder Ende des Druckes, ein approbatio genannter Vermerk über eine Begutachtung durch ein Zensurorgan, das den Druck freigegeben hat (Nuovo 2015: 195-257). ${ }^{9}$

Die unterschiedlichen, soeben skizzierten Formen der frühneuzeitlichen Zensur sollen in den folgenden Abschnitten für die Sphaera-Tradition untersucht werden. Hierbei steht die jeweilige Zensur der römischen, spanischen und portugiesischen Inquisition im Mittelpunkt, wobei stichprobenartig auch weitere, lokale Zensurdokumente miteinbezogen werden. ${ }^{10}$ Die römischen und iberischen Indices stellen die wichtigsten Quellen dieser Untersuchung dar und sind für das 16. Jahrhundert in den von Jesús Martinez de Bujanda besorgten Editionen gut erschließbar (Bujanda et al. 1984-2002 = ILI). Für die Indices des 17. Jahrhunderts werden die zeitgenössischen Drucke herangezogen (Mascarenhas 1624; Zapata 1632; Sotomayor 1667). Interne Akten der römischen Zensur (insbesondere aus dem römischen Archivio della Congregazione per la Dottrina della Fede) sind über die bereits erwähnte Edition von Leen Spruit und Ugo Baldini für das 16. Jahrhundert ebenfalls zugänglich (Baldini \& Spruit 2009= CCMS). Darüber zeitlich und räumlich hinausgehende Akten und Archivalien werden allerdings nur in Einzelfällen berücksichtigt - gerade für die Arbeit der iberischen Zensoren fehlen umfassende Editionen bis heute. Die wichtigsten der im Folgenden herangezogenen Dokumente und die darin berücksichtigten Sphaera-Drucke werden am Ende dieses Aufsatzes in einem Anhang tabellarisch (Tab. 1, 2 und 3) dargestellt.

\section{Früheste Sacrobosco-Zensuren und die Installation des Index}

Johannes de Sacroboscos Tractatus de sphaera stand im Mittelalter nicht im Fadenkreuz der Inquisition oder anderer Zensurorgane, enthielt sein Werk ja auch kaum theologisch Relevantes und sparte auch den seit den Kirchenvätern problematisierten Aspekt der juridischen Astrologie gänzlich aus. ${ }^{11}$ Den vermutlich ersten Fall in der Zensurgeschichte der Sphaera-Tradition bildete Cecco d'Ascolis (c. 1257-1327) vor 1324 verfasster Kommentar zu Sacroboscos Werk (Thorndike 1949: 343-410). In diesem Kommentar beschäftigte sich Cecco nämlich umfassend mit Astrologie, Magie und illegitimen Einzelkünsten. Seine Verurteilungen von 1324 und 1327 durch die Inquisition sind wohl zumindest teilweise auch auf seinen Sphaera-Kommentar zurückzuführen. ${ }^{12}$ Dieser Kommentar wurde zwischen 1499 und 1518 jedoch viermal gedruckt, ohne dass diese Ausgaben bei Zensurbehörden Anstoß gefunden hätten. ${ }^{13}$ 
Zensuren der frühen Druckgeschichte der Sphaera-Tradition lassen sich ohnedies kaum finden. Einzig ein Frontispiz eines Sphaera-Drucks von 1488, das die barbusige Urania allegorisch darstellt, wurde in einer einzelnen Ausgabe offenbar zensiert: Die nackten Brüste wurden entfernt (Cirella \& Rinaldi 2015).${ }^{14}$ Eine solche Zensur betrifft jedoch weder den Text oder Autor des Werks, noch war sie Teil einer systematischen Bildkorrektur, sondern geht wohl auf das Schamgefühl des unbekannten Vorbesitzers des Exemplars zurück, das heute in Capodimonte liegt.

Die eigentliche Zensurgeschichte der gedruckten Sphaera-Tradition beginnt mit der Vorarbeit am römischen Index von 1559, den Papst Paul IV (1476-1559) herausgab. Schon vorher waren Autoren, die auch SphaeraTraktate verfasst hatten, in das Visier der Inquisition geraten, doch ausschließlich aufgrund ihrer theologischen oder politischen Werke (Del Col 1980; Barbieri 2011). Eine Erwähnung, geschweige denn eine Zensur einzelner Sphaera-Traktate in den edierten Zensurdokumenten aus der Zeit vor 1558 findet sich hingegen nirgends. Der Paulinische Index von 1559 zeichnete sich seinerseits vor allem dadurch aus, sämtliche Werke einer großen Anzahl (ca. 550) von - meist protestantischen - Autoren zu verdammen, ohne diese Werke jedoch stets im Einzelnen aufzuführen. Dass die Kommission, die mit der Etablierung der Ketzerliste betraut war, auch Sphaera-Traktate durchblätterte, um einzelne Autoren $\mathrm{zu}$ inkriminieren, dokumentiert eine anonyme handschriftlich hinterlassene Quelle aus der römischen Kommission, die als eine Art interne Vorarbeit für den Index zu betrachten ist (CCMS I, 132, 134). ${ }^{15}$

Zunächst wird in dem genannten handschriftlichen Dokument Philipp Melanchthons (1497-1560) Schwiegersohn Kaspar Peucer (1525-1602) genannt, der nicht nur als Anhänger (sequax) des Reformators, sondern auch als Autor des Werkes Elementa doctrinae de circulis coelestibus (1551) identifiziert wird, das er dem lutherischen Herzog von Sachsen Augustus (1526-1586) gewidmet habe. ${ }^{16}$ Hartmann Beyer (1516-1577) wird in diesem Dokument ebenfalls von den Zensoren belastet, weil er im Widmungsbrief seiner Quaestiones novae in libellum de sphaera Ioannis de Sacro Busto (1549) bekennt bei Melanchthon studiert zu haben. ${ }^{17}$ Des Weiteren wird Jakob Milich (1501-1559) in Melanchthons Brief in der 1531 in Wittenberg gedruckten Sacrobosco-Ausgabe, die weiter unten noch näher besprochen werden soll, lobend erwähnt, was diesem Gelehrten von katholischer Seite nicht zum Vorteil gereichen konnte und er somit ebenfalls von den römischen Zensoren genannt wurde. ${ }^{18}$ Auf Grundlage solcher Publikationen, die ausnahmslos aus dem Kreis der lutherischen Wittenberger Universität stammten und als Sphaera-Traktate gelten können, konnten die Zensoren somit drei Autoren als Melanchthon-Anhänger und damit als Häretiker 
identifizieren, deren Werke 1559 sämtlich zensiert wurden (ILI VIII, 391, 489, 512-513; Brosseder 2004: 95, 138, 144, 278, 308).

\section{Sphaera-Traktate auf dem Antwerpener Expurgationsindex von 1571}

Schon der Tridentinische Index von 1564 entschärfte in manchen Hinsichten die rigide opera-omnia-Leitschnur von 1559, die Katholiken letztlich auch das Lesen theologisch unbedenklicher Werke unmöglich machte, sofern ihre Autoren als Häretiker galten. Etwaige Sphaera-Traktate wurden 1564 jedoch nicht ausdrücklich ausgenommen. Dies belegt hingegen der Antwerpener Expurgationsindex, der 1571 federführend vom Spanier Benito Arias Montano (1527-1598) herausgeben wurde (Rekers 1961; Eisenstein 2005: 332). Dieser Index wurde in späteren italienischen und iberischen Indices berücksichtigt und verdient daher an dieser Stelle eine kurze Erwähnung.

Im Antwerpener Index wurden beispielsweise Peucers Elementa prinzipiell erlaubt, solange eine knappe, belanglos erscheinende, womöglich sogar missverstandene Passage aus dem Widmungsschreiben an den Fürsten gestrichen werde (ILI VII, 781; Montana 1571: 55). ${ }^{19}$ Anders als in vorangehenden Indices wurde hier also Peucer zwar als Ketzer erwähnt, einzelne seiner Schriften, darunter auch seine Elementa, wurden jedoch nach Expurgation erlaubt. Erasmus Oswald Schreckenfuchs (1511-1575), ebenfalls Protestant und Schüler Sebastian Münsters (1488-1552), den schon der Index aus Leuven 1546 führte, war auch im Paulinischen Index von 1559 erwähnt worden (ILI II, 151; VIII, 446). 1571 wurde auch Schreckenfuchs' Sacrobosco-Kommentar (1569) verzeichnet, allerdings ausdrücklich als unbedenklich befunden (nihil continet, quod offendat pietatem vel bonos mores). ${ }^{20}$ Erstmals in einem Index genannt wurde Sebastian Theodoricus (Dietrich; c. 1520-1574), dessen Quastionenkommentar zu Sacrobosco (1564) allerdings gleichfalls genehmigt wurde (ILI VII, 782; Montana 1571: 56). ${ }^{21}$

Diese drei Beispiele zeigen also bereits einen Kurswechsel an: Protestantische Autoren mögen Ketzer sein, die Lektüre ihrer Werke zu nicht-theologischen Themen sollte jedoch auch Katholiken gestattet sein - zumindest in den Augen der flämischen Indexkommission. Die zweite der zehn Grundsatzregeln der Tridentinischen Buchzensur, die vorschrieb, dass solche Werke der Häretiker ohne theologische Bewandtnis erst nach Prüfung seitens des Bischofs oder des Inquisitors von Katholiken gelesen werden durften, wurde 1571 mitgedruckt und in den erwähnten Fällen umgesetzt, 
insofern keine individuelle Sondererlaubnis mehr nötig war, da die Expurgationsanweisung mitgegeben wurde. ${ }^{22}$ Ebenso fielen die drei genannten Werke nicht unter die neunte Regel, die, vereinfacht gesagt, Werke zu Magie und Astrologie verbot; die genannten Schriften wurden in der Nomenklatur der Zensoren von 1571 nämlich unter den libri mathematici geführt (Baldini 2001: 90). Zudem bediente sich der Antwerpener Index im Gegensatz zu den beiden erwähnten römischen Indices des Mittels der Expurgation, um einzelne Werke zu korrigieren statt diese pauschal zu verbieten (Spruit 2010: 180). ${ }^{23}$

\section{Der Fall „Mauro da Firenze"}

Doch auch der römische Index wurde umfangreicher und schloss einen weiteren Sphaera-Traktat ein, der genauere Betrachtung verdient, da sich diese Zensur durch kein offensichtlich gegenreformatorisches oder theologisches Kalkül begründen lässt. In zahlreichen kürzeren und lokalen Indices aus der Zeit zwischen 1576 und 1587 findet sich, meist unter der Rubrik sogenannter „verdächtiger italienischer Bücher“, folgende Angabe eines Buches: „Dichiaratione delle sphere.“24 Ein Autor wird nicht genannt, die Schrift wird meist auf 1555 datiert und als Buch im Oktavformat beschrieben. ${ }^{25}$ Es ist zu vermuten, dass auf die Schrift Annotationi sopra la lettione della Spera de Sacro Bosco des Florentiner Serviten Marco Mauro da Firenze (1493-1556) verwiesen wird (Mauro da Firenze \& Sacrobosco 1550; Pereira da Silva 1915: 42; Bennett \& Meli 1994: 36-39; Gentile und Gilly 2001: 293-296; ILI IX, 115). ${ }^{26}$ Gegen diese Identifizierung spricht allerdings, dass dieses Werk höchstwahrscheinlich nur 1550, nicht 1555 in Florenz erschienen ist und Quartformat aufweist. Gestützt wird die $\mathrm{Zu}$ schreibung hingegen dadurch, dass Mauros Werk - nun eindeutig in Titel und Autor benannt - auf dem vom Generalinquisitor Gaspar de Quiroga y Vela (1512-1595) organisierten spanischen Index von 1583 sowie auf den römischen Indices von 1590 und 1593 in die Liste der verbotenen Büchern aufgenommen wurde (ILI VI, 644-645, 658; IX, 388). Expurgationsrichtlinien oder Gründe der Zensur fehlen in allen Indices und über den Serviten Mauro ist biographisch zu wenig bekannt, um die Indizierung seines Werkes hieraus fundiert abzuleiten. Zudem hatte Mauro bereits 1537 eine italienische kommentierte Übersetzung von Sacroboscos Sphaera in Venedig publiziert, die in den Indices nirgends erwähnt wird und daher vermutlich entweder als unbedenklich erachtet oder gar nicht erst geprüft wurde (Mauro da Firenze \& Sacrobosco 1537). ${ }^{27}$ Seine Annotationi weisen jedoch inhaltliche Merkmale auf, die das Verbot des Titels motiviert haben 
dürften. Eine kursorische Beschreibung des Werkes scheint daher sinnvoll, wenngleich genauere Analysen zukünftiger Forschung vorbehalten bleiben.

Die Annotationi sind nur auf den ersten Blick ein bloßer Kommentar zu Sacrobosco. Tatsächlich enthält der Band mindestens sechs recht eigenständige Werke und ebenso viele Widmungsbriefe und Paratexte. ${ }^{28}$ Der eigentliche Kommentar zu Sacrobosco (5-93), der auf Italienisch verfasst ist, aber auf den lateinischen Text der Sphaera Bezug nimmt, geht wohl auf eine Vorlesung zurück, die Mauro vor einigen Aristokraten gehalten hat. Sie ist weitestgehend propädeutisch ausgerichtet und bietet eine Einführung in notwendige mathematische und geometrische Grundlagenkenntnisse. Gleichzeitig beschäftigt sich Mauro dort auch mit instrumentellen astronomischen Messungen (72-79). Verweise auf Messungen aus Florenz (71, 76, 83, 88) sowie auf das Jahr 1546 (62) dürften Ort und Zeit der Entstehung des Textes markieren. Widmungen an den Medicifürsten Cosimo (1519-1574) datieren von 1546 (156-157) beziehungsweise 1547 (3-4). In seinem Kommentar weist Mauro auch auf Fehler seiner kommentierten Übersetzung von 1537 hin (54). Vielleicht fertigte er aus diesem Grund eine neue Übersetzung der Sphaera an (97-146), die im Druck von 1550 ebenfalls enthalten ist.

Mauros Kommentar erschien aus Sicht der Zensur vielleicht deshalb verdächtig, weil es dort auch um Astrologie geht (22), die unter bestimmten Bedingungen seit 1557 Indizierungsgrund war. Jedoch wird in Mauros Werk mantische und weissagende Astrologie nicht verfolgt und vielmehr der praktische Wert der natürlichen Astrologie für Kontexte wie Landwirtschaft und Medizin betont (35) - schon im Index von 1559 wurde Astrologie im Kontext dieser praktischen Zusammenhänge ausdrücklich geduldet (ILI VIII, 775). Somit mag auch das auf der Titelseite der Ausgabe abgedruckte Horoskop einen irreführenden Eindruck hinterlassen haben, denn um Horoskope geht es in Mauros Text nirgends direkt. ${ }^{29}$

Ein weitaus wahrscheinlicher Grund für das Verbot der Indexkommission ist jedoch die starke theologische Ausrichtung des Werks, zumal Mauro selbst Magister der Theologie war. Diese Tendenz zeichnet sich bereits in zwei kurzen im Band enthaltenen „mathematischen Übungen“ (94-96, 147-153) ab und wird in einem eigenen Teil, der „Spera theologica et divina" genannt wird, ausführlicher entwickelt. Ausgehend von platonischen Ansätzen wird spekulativ eine "theologische Sphäre“ entworfen, wie sie schon prominent in den Werken Nikolaus von Kues zu finden ist (Müller 2011). Dieser platonische Einschlag - Mauro bebildert sogar die „Sphaera platonica“ (96) - drückt sich auch in der Auswahl der Autoritäten aus, die er zitiert: Cusanus $(47,149,163,166,170,173,174,175,180,181,183,186$, 187, 190, 193, 199), Plato und die Platoniker (95, 147, 148, 162, 175, 179, 190, 193), Marsilio Ficino (95), Hermes Trismegistos (182), (Pseudo-)Dio- 
nysius Areopagita $(88,166,181)$ sowie der Kirchenvater Augustinus (94, 151, 165, 167, 170, 173, 178, 180, 183, 185, 186, 187, 188, 196) bilden hier die wichtigsten Referenzen. Verweise zu Autoren der Astronomiegeschichte oder der klassischen und mittelalterlichen Theologie und Philosophie finden sich deutlich seltener, sieht man von Erwähnungen Sacroboscos oder Aristoteles' ab. ${ }^{30}$ Unter Zeitgenossen erwähnt Mauro nur Alessandro Piccolomini (1508-1578) (35, 54), der 1540 seine italienische Sfera del Mondo publiziert hatte, sowie den Lutheranhänger Johannes Bernhardi „Velcurio“ (c. 1490-1534) (192). ${ }^{31}$ Auffallend ist jedoch auch die große Anzahl zitierter lateinischer Bibelzitate und Schriftverweise, insbesondere aus dem Neuen Testament. ${ }^{32}$

Die Themen seiner „Spera Theologica“ umfassen somit auch wichtige Theologoumena: Trinität (173-181), Inkarnation (181-186), Eucharistie (186-189), Unsterblichkeit der Seele (190-197), Willensfreiheit und Gnadenlehre (194-197). ${ }^{33}$ Insbesondere in Bezug auf die Unsterblichkeit der Seele und die Willensfreiheit vertritt Mauro in den Annotationi Positionen, die im Italien des 16 . Jahrhunderts als riskant zu bewerten sind. ${ }^{34}$ Mauro schien dies geahnt zu haben und holte sich daher 1548 die theologische Einschätzung des Minoriten Raffaello Sannino ein (154). Der Konsultierte bewertete das Werk hingegen als „sehr nützlich“ und als „approbiert“ (155). Die „Spera Theologica“ widmete Mauro nicht direkt Cosimo de' Medici, sondern dessen Frankreichgesandten Bartholomeo Panciatichi (1507-1582), der jedoch mit dem Protestantismus anbändelte und 1551 deshalb auch verhaftet wurde (Caravale 2014). Es scheint daher wahrscheinlich, dass die Behandlung theologischer Themen, das in diesem $\mathrm{Zu}$ sammenhang erfolgte Vertreten streitbarer Positionen sowie die Sympathiebekundung gegenüber einem überführten, wenngleich später begnadigten Häretiker, Mauros Werk in den Augen der Indexkommission verdächtig erscheinen ließ und damit verboten wurde.

Obgleich Mauros Werk vermutlich nach 1550 nicht nachgedruckt wurde - Exemplare eines Nachdrucks sind zumindest nicht bekannt - wurde die Schrift um 1633 in einer recht aufwändigen Handschrift von Matías de los Reyes ins Spanische übersetzt und dem spanischen Adligen Luis Ramón de Aragón y Fernández de Córdoba $(+1642)$ gewidmet. ${ }^{35}$ Diese Übersetzung, die an einigen Stellen vom italienischen Original abweicht und dieses mitunter deutlich erweitert, betrifft jedoch nur den ersten Teil von Mauros Werk und lässt die theologisch-spekulativen Traktate aus. Deutlich mehr Aufmerksamkeit erhält dort die Astrologie des Tierkreises, die als Addicion immerhin 20 Seiten umfasst (72r-82v) und in Mauros Annotationi keine Vorlage hat. Der womöglich höfische Entstehungskontext der Handschrift konnte diese unter Adeligen beliebte Thematik eventuell an der Buchzensur vorbeiführen. 


\section{Sphaera-Traktate in den iberischen Indices}

Mauros Annotationi wurden im römischen Index von 1590, der von Papst Sixtus V (1521-1590) herausgegeben wurde, zwar aufgeführt und so auch noch in den vorläufigen Index seines Nachfolgers Clemens VIII (1536-1605) mit aufgenommen, doch in der endgültigen Version des römischen Indexes, der 1596 publiziert und erst 1664 erneuert wurde, fehlt Mauros Werk unter den verbotenen Titeln - wie auch alle anderen Sphaera-Traktate. ${ }^{36}$ Zwar hatte schon 1587 ein anonymer Mitarbeiter der römischen Indexkongregation in einer handschriftlichen Liste $\mathrm{zu}$ expurgierender libri mathematici auch die Sphaera-Traktate der ketzerischen Autoren Peucer, Schreckenfuchs, Theodoricus, Antonio Bruccioli (c. 1498-1566) und Melanchthon vermerkt. ${ }^{37}$ Eine solche Expurgation erfolgte in Rom jedoch nicht.

Eine deutliche Erweiterung der Liste verbotener Sphaera-Traktate bezeugen hingegen die spanischen und portugiesischen Indices. Nach dem Quiroga-Index von 1583 finden sich Mauros Annotationi in den spanischen Indices von 1612, 1632 und 1640, die von den spanischen Generalinquisitoren Bernardo de Sandoval y Rojas (1546-1618), Antonio Zapata (1550-1635) und Antonio Sotomayor (1548-1648) herausgegeben wurden, sowie im portugiesischen Index, den Generalinquisitor Fernando Martins Mascarenhas (1548-1628) 1624 besorgte (Sandoval y Rojas 1612: 77; Mascarenhas 1624: 158; Zapata 1632: 763; Sotomayor 1667: 795)..$^{38}$

1612 wurde zudem erstmals eine bibliographische Angabe aus der erweiterten Fassung von Conrad Gessners (1516-1565) Bibliotheca (1583) zensiert: ${ }^{39}$ Dort wird auf die bereits erwähnte Wittenberger Ausgabe der Sphaera sowie von Sacroboscos Computus ecclesiasticus hingewiesen, der ebenfalls mit einem Vorwort Melanchthons versehen war (Knobloch \& Reich 2004; Rosen 1974). Diese Stelle musste aus Gessners Werk gestrichen werden. Eine solche Edition von 1563, die beide genannten Texte Sacroboscos enthielt, wurde jedoch erst 1624 im portugiesischen Index inspiziert und expurgiert. ${ }^{40}$ Die dort zu lesenden Anweisungen lauten: Melanchthons Name sei in seinen Vorworten zur Sphaera und zum Computus zu entfernen. In jener kalendarischen Schrift, die 1538 erstmals in Wittenberg zusammen mit der astronomischen Schrift gedruckt wurde, musste sogar ein Passus gestrichen werden, da sich dieser mit den sogenannten „ägyptischen Tagen" beschäftigte, einer astrologischen Theorie besonders unglücksaffiner Tage, die schon von Kirchenvätern als heidnischer Aberglaube kritisiert worden war (Skemer 2010; Harmening 1979: 165). Zudem bemerkte der Zensor, dass die Sphaera-Bände regelrechte Kompendien darstellten und etwa auch ein Sphaera-Traktat Giovanni Pietro Pierio Valerianos (1477-1558) und ein knapper Traktat über Sonnen- und Mondfinsternisse 
enthielt, der dem antiken Philosophen Proklos (412-485) zugeschrieben wurde. ${ }^{41}$ Letzterer Traktat solle gänzlich expurgiert werden. Zwar werden die Gründe hierfür nicht genannt, doch vermutlich motivierte der astrologische Ansatz der knappen Schrift diese Maßnahme (Siorvanes 2014: 49). Zudem zeigt sich hieran implizit, dass nicht nur Wittenberger Auflagen dieser Sphaera-Bände begutachtet wurden, da Sacroboscos Sphaera zusammen mit seinem Computus, Valerianos Compendium und Proklos' Traktat ledigleich 1573 in Antwerpen verlegt wurde, dort jedoch Melanchthons Vorwort zum Computus wiederum fehlte. ${ }^{42}$ Die Zensuren betrafen also eine nicht genau bestimmte Kombination verschiedener SphaeraBände und nicht eine einzige Ausgabe, geschweige denn die genannte aus Wittenberg von 1563.

1624 wurden zusätzlich noch zwei weitere Sphaera-Traktate expurgiert, die zwar beide nicht direkt auf Sacroboscos Sphaera zurückgreifen, dieser aber in Thematik und Aufbau gleichen: Pedro de la Hera y de la Varras Reportorio del mundo particular de las Spheras del Cielo y Orbes elementales (1584) und George Buchanans (1506-1582) Lehrgedicht Sphaera (1587) (Mascarenhas 1624: 274, 596; Buchanan 1587; Hera y de la Varra 1584). Die Zensur Buchanans ist klar durch dessen konfessionelle Angehörigkeit zu erklären, war der Schotte ja schon 1537 wegen Ketzerei verurteilt worden. Daher sollte auch sein Name auf der Titelseite um rühmende Prädikate gekürzt und um den Zusatz „verurteilter Autor“ erweitert werden. Zudem war ein Teil des Vorwortes wegen der Nennung anderer in Ungnade Gefallener zu kürzen sowie drei Widmungsgedichte zu entfernen, die von den Häretikern Johannes Pincier d. J. (1521-1591), John Johnson (1500-1594) und Rudolf Goclenius d. Ä. (1547-1628) verfasst worden waren. Varras Reportorio wiederum musste wegen bestimmter Aspekte vorhersagender Astrologie expurgiert werden, wie ausdrücklich unter Berufung auf die Vorschriften des sixtinischen Indexes (1590) erklärt wurde. ${ }^{43}$ Varras und Buchanans Werke wurden jedoch nach der eher geringfügigen Korrektur freigegeben.

In den spanischen Indices von Zapata (1632) und Sotomayor (1640) wurden die genannten Expurgationen von 1624 übernommen (Zapata 1632: 105-106, 407, 693, 763; Sotomayor 1667: 115, 433, 723, 795). 1632 wurde auch Schreckenfuchs' Sphaera wieder aufgeführt, jedoch anders als $1571 \mathrm{im}$ Index aus Antwerpen bis zur ausstehenden Expurgation verboten (Zapata 1632: 345). Auch Peucers astronomische Werke wurden pauschal unter der Bedingung einer nicht explizierten Expurgation freigegeben (Zapata 1632: 169). 1640 wurde zumindest Schreckenfuchs Werk nicht mehr aufgeführt (Sotomayor 1667: 366). Dafür tauchten nun Sphaera-Traktate deutscher protestantischer Autoren wie Nikolaus Selnecker (1532-1592), Jakob Ziegler (1470-1549), Hartmann Beyer und Wilhelm Adolf Scribonius 
(1550-1600) auf (Sotomayor 1667: 6, 479, 546, 805). ${ }^{44}$ Die astronomischen Werke dieser Autoren wurden zwar genehmigt, allerdings mit der Auflage stets im Buch zu vermerken, dass es sich bei ihren Verfassern um „auctores damnati" handelte. Die iberischen Zensurbeamten kannten also eine umfassende Menge der Sphaera-Traktate des 16. Jahrhunderts, insbesondere aus deutschen Gebieten. Anders als vielleicht zu erwarten, schütteten sie jedoch nicht das Kind mit dem Bade aus: Mochten die Autoren auch Ketzer sein, ihre weltlichen Schriften waren hilfreich und durften, nach Anwendung etwaiger Expurgationen, auch von Katholiken gelesen werden.

\section{Die censura praevia und licentiae}

Die bisher beschriebenen Fälle schildern die Zensur gewissermaßen aus der Top-down-Perspektive der Zensoren. Doch inwieweit wurden die Vorschriften umgesetzt? Wie betraf die Zensurpolitik der katholischen Behörden die Besitzer, Leser und Autoren der Bücher? Auch hierzu lassen sich am Fallbeispiel der Sphaera-Traktate einige Beobachtungen anführen, die zwar nicht zu verallgemeinern sind, jedoch ein recht breites Spektrum des möglichen Einflusses der Zensur kartieren.

Für die Autoren und mitunter Verleger der Sphaera-Traktate waren die Bestimmungen der Zensur durchaus relevant, insofern die Bände vor dem Druck durch die Inquisition oder den „Magister Sacri Palatii“ auf Unbedenklichkeit hin zu prüfen waren (Nuovo 2015: 237-244). Es sind jedoch keine Dokumente bekannt, die diese Praxis speziell für Sphaera-Traktate belegen. ${ }^{45}$ Und auch nur in einer relativ geringen Anzahl der SphaeraDrucke ist zu Beginn oder Ende der Bände eine inhaltliche Approbation mit abgedruckt, die eine präventive Inspektion durch ein Zensurorgan impliziert.

Bei den 17 identifizierten Fällen solcher Vorabzensuren (censurae praeviae), die im Anhang dieses Aufsatzes tabellarisch zusammengestellt sind (Tab. 3), scheinen mehrere Beobachtungen erwähnenswert: Der Publikation von Christoph Clavius' (1538-1612) Sphaera-Kommentar (1570) ging eine interne Zensur voraus, wie dies für jede jesuitische Publikation erforderlich war (Baldini 1985; Lattis 1989: 64-65). ${ }^{46}$ Doch nur in acht der 22 Clavius-Ausgaben ist eine ausdrückliche Genehmigung eines Zensors abgedruckt worden. ${ }^{47}$ Nimmt man die Clavius-Ausgaben aus, ist von den verbleibenden neun vorab zensierten Sphaera-Drucken nur eine einzige lateinische dabei. ${ }^{48}$ Einer Vorabzensur wurden also vor allem volkssprachige Sphaera-Traktate unterzogen (Crowther et al. 2015). Regional dominiert der iberische Raum (sechs Drucke), gefolgt von italienischen Städten (drei 
Drucke). In Frankreich, den Niederlanden oder deutschsprachigen Gebieten, so der Umkehrschluss, griffen die katholischen Bestimmungen der censura praevia somit nicht, da diese ja außerhalb der Jurisdiktion von römischer oder iberischer Inquisition lagen. Betrachtet man die Autoren der untersuchten Druckgenehmigungen, sind diese in der Regel Mitarbeiter der Inquisition, in manchen Fällen jedoch auch Professoren der Astrologie oder der Mathematik. ${ }^{49}$

Auch die Besitzer von Bibliotheken in Italien waren von der römischen Zensurpolitik betroffen: Zur Kontrolle der Bibliotheken und der Erweiterung des Indexes sah die römische Inquisition vor, einen census aller Buchbestände zu erstellen. Insbesondere die religiösen Orden in Italien kamen diesem Auftrag nach und verfassten zunächst Listen von solchen Büchern, die oder deren Autoren bereits auf einem Index standen (Bruni 2013). Um zensierte Bücher jedoch weiterhin legitim besitzen und lesen zu können, bedurfte es eines förmlichen Antrags sowie ausdrücklicher Genehmigungen durch die römische Inquisition, die sogenannte licentiae erteilte. Diese Lizenzen wurden meist in Form von Listen für suspekte oder verbotene Titel in einem Bibliotheksbestand ausgestellt. Erhaltene Exemplare solcher Antrags- oder Genehmigungslisten aus dem 16. Jahrhundert zeigen, dass vielfach Sphaera-Traktate aufgeführt wurden, welche die Inquisition als "gefährlich“ oder „verboten“ einstufte.

Auf einer solchen zwischen 1560 und 1580 entstandenen Liste, mit der Ortensio Albertoni die licentiae für die verbotenen astrologisch-astronomischen Schriften in der Bibliothek seines Vaters Giovanni anfragte, taucht etwa eine Sphaera Sacroboscos auf, die zusammen mit einer Ephemeride für das Jahr 1532 des Astrologen Luca Gauricos (1476-1558) in einem Band gebunden war (CCMS III, 2610). ${ }^{50}$ Auf einer anderen Liste von 1596, die eine Leseerlaubnis für die verdächtigen Bücher im Besitz von Guido Cavalcanti beantragt, finden sich Mauros Annotationi sowie ein umfangreicher, auch astrologisch ausgerichteter Kommentar zur Sphaera aus der Feder des Theologen Francesco Giuntini (1523-1590) (CCMS III, 2692). ${ }^{51}$ 1598 wurden der römischen Bibliothek des Oratoriums der Kirche Santa Maria in Vallicella wiederum gleich eine ganze Reihe von Lizenzen für Sphaera-Bände erteilt, darunter in Venedig, Wittenberg und Paris gedruckte Ausgaben mit Melanchthons Vorwort sowie Pietro Catenas (1501-1577) Sphaera (1561) (CCMS III, 2723). ${ }^{52}$ Diese Dokumente der römischen Inquisition geben also Zeugnis davon, dass die Buchbestände kritisch erfasst wurden sowie ihre Besitzer achtsam Lese- und Besitzerlaubnisse einholten; inter alia betraf dies auch Sphaera-Bände.

Wie sich einzelne Leser gegenüber den Vorgaben des spanischen Indexes verhielten, lässt sich schwerer rekonstruieren, doch auch hier findet sich zumindest ein Beispiel, das die Sphaera-Tradition berücksichtigt. In 
Reaktion auf den spanischen Index von 1583, der Schriften bekannter Häretiker verbot, versuchte 1585 Jerónimo de Guzmán gegenüber der Inquisition unter anderem die Freigabe eines Sphaera-Traktats des Protestanten Victorinus Strigel (1524-1569) (Epitome doctrinae de primo motu) zu erwirken, dessen Werke ohne Genehmigung nicht gelesen werden durften (Pardo Tomás 1991: 285). Er berief sich hierbei auf die dritte der tridentinischen Indexregeln, die besagte, dass Übersetzungen von Ketzern, die nicht das Thema „Religion“ betreffen, durchaus genehmigt werden können. Zwar handelte es sich bei Strigels Werk nicht um eine Übersetzung, aber um eine Schrift ohne theologische Relevanz. Doch es scheint, dass Guzmáns Gesuch insofern unerhört blieb, als dass das genannte Werke Strigels im folgenden Index von 1612 weiterhin verboten war (Sandoval y Rojas 1612: 98, 735). ${ }^{53}$

\section{Expurgationen in Sphaera-Drucken}

Doch auch wenn ein Buch einmal gedruckt und in Umlauf war, endete die eingreifende Zensur nicht. Inwieweit sämtliche von den Indices geforderten Expurgationen in den Sphaera-Bänden tatsächlich umgesetzt wurden, lässt sicht nicht sagen. Ein vergleichsweise gut dokumentiertes Beispiel der Expurgationspraxis ist jedoch die bereits mehrfach erwähnte Wittenberger Sphaera-Edition von 1531, die offenbar im Kreis um Philipp Melanchthon erstellt wurde und dessen Widmungsschreiben an Simon Grynaeus (1493-1541) enthält, in dem er den Nutzen der Astrologie für die christliche Lehre hervorhebt (Pantin 1987; Lalla 2003; Knobloch \& Reich 2004; Melanchthon 2003: 163-171). Bereits ein Jahr nach Erscheinen dieser Ausgabe wurde sie in Venedig von Melchior Sessa († c. 1565) nachgedruckt. ${ }^{54}$ Melanchthon selbst hatte Venedig zwar nicht bereist, erfreute sich aber in manchen italienischen Kreisen, vermutlich auch im liberalen Venedig, einer gewissen Anerkennung (Rhein 1996; Caponetto 2000). Dies spiegelt sich auch darin wider, dass andere Bände mit Beteiligung Melanchthons im Laufe der 1530er Jahre in Venedig von Sessa gedruckt wurden. ${ }^{55}$ Der Kontaktpunkt zwischen Wittenberg und Venedig, das heißt, die Antwort auf die Frage, wie Sessa an die Wittenberger Ausgaben gelangte, bleibt jedoch offen. Dass sie auf ihrem Weg südlich über die Alpen in katholische Gebiete der Inquisition umso leichter in die Hände fallen dürfte, scheint hingegen naheliegend.

Schon Isabelle Pantin hat argumentiert, Melanchthons Astrologievorwort sei „probablement moins remarquable par sa teneur même que par le destin qu'elle a connu“ (Pantin 1987: 85). Wesentliche Etappen haben Pan- 
tin und andere bereits herausgearbeitet: Zwischen 1531 und 1629 sind über 60 Drucke zu identifizieren, die Melanchthons Brief enthalten. Er wurde mitunter auf 1540 umdatiert, gekürzt, anonymisiert und sogar übersetzt. Man findet ihn in Sphaera-Sammelbänden aus Druckerpressen in Wittenberg, Paris, Köln, Antwerpen, Lyon und Venedig, die nicht nur klassische Werke oder Ausschnitte zur Astronomie enthalten, sondern auch Valerianos Compendium sowie Sphaera-Kommentare von Élie Vinet und Francesco Giuntini. Der katholische Kölner Theologe Albertus Hero (1549-1589) kombinierte den Brief, allerdings in anonymer Form, sogar mit Auszügen aus Christoph Clavius' Kommentar.

Die Forschung hat ebenfalls schon herausgestellt, dass sich viele Ausgaben finden, in denen der Brief oder Melanchthons Name nachträglich entfernt wurde (Oosterhoff 2015: 20-21; Gingerich 1988: 270; Pantin 1987: 95; Crowther et al. 2015: 10). Diese Praxis, die zahlreich vorhandenen Briefe oder Vorworte Melanchthons aus solchen Schriften, die ihrem Gegenstand nach die Theologie nicht betreffen, herauszulösen beziehungsweise Melanchthons Namen zu streichen, wurde bereits im Index von Portugal (1561) gefordert, der vom zukünftigen Sekretär der tridentinischen Indexkommission, Francisco Foreiro (1523-1581), erstellt wurde (ILI IV, 1000). ${ }^{56}$ Auch die Arbeit der römischen Indexkongregation belegt diese Praxis in vielen Anweisungen (CCMS II, 1411; III, 2334, 2336, 2338-9). In der Mehrzahl der Ausgaben wurde der Brief nicht entfernt, sondern verschiedene seiner paratextuellen Bestandteile geschwärzt, etwa die $\mathrm{Na}$ men "Melanchthon" und „Grynaeus" sowie der Ortsname „Wittenberg“.57 Diese Ergebnisse der Expurgation finden sich auch in Exemplaren aus den Beständen von Klöstern und katholischen Kollegien - die sehr wohl ihre Melanchthon-Ausgabe besaßen, dessen Namen aber entfernten. ${ }^{58}$

Doch Melanchthon und Grynaeus waren nicht die einzigen Ziele katholischer Expurgationen. Richard J. Oosterhoff hat etwa darauf hingewiesen, dass in einem Sphaera-Band aus der Huntington Library der Name des mittelalterlichen Philosophen, Kosmologen und Klerikers Pierre d'Aillys (1350-1420) sorgfältig aus dem kompletten Exemplar entfernt wurde (Oosterhoff 2015: 21). ${ }^{59}$ Oosterhoff schließt zu Recht, „censorship mostly extends only to names, not text itself“. Auch Melanchthons Vorwort war nicht etwa problematisch, weil es von Astrologie im Dienste der christlichen Lehre handelte, sondern weil es von einem Ketzer verfasst worden war. Diese nicht-inhaltlichen Zensurspuren sind jedoch deshalb nicht weniger interessant und aussagekräftig. Sucht man etwa nach anderen geschwärzten Personennamen, mag es wenig überraschen, dass der von Desiderius Erasmus mehrfach unleserlich gemacht worden war. ${ }^{60}$ Etwas überraschender ist es hingegen, dass Giuntinis Name von einer Titelseite entfernt wurde, da seine Werke nie auf den Index gesetzt wurden. Allerdings ist sein 
Sphaera-Kommentar auf der Liste verdächtiger Titel im Besitz von Guido Cavalcanti zu finden, und die Inquisition prozessierte mehrfach gegen den Astrologen. ${ }^{61}$

In Francesco Barozzis (1537-1604) Vorwort zu seiner Cosmographia (1585) wiederum schwärzte ein Zensor eindringlich den Namen eines gewissen Menelaos (Barozzi 1585: B5v). ${ }^{62}$ Zwar meint Barrozzi hiermit zweifelsohne den hellenistischen Mathematiker (c. 45-c. 110) mit diesem Namen, dessen Werk Sphaerica durchaus bekannt war. Doch vermutlich dachte der Zensor seinerseits an den gleichnamigen, fehlgeleiteten Hohepriester der Diadochenzeit, der im Alten Testament erwähnt wird (Dowden 1999). Diese Expurgation ist aus zwei Gründen interessant. Zum einen ließ der Zensor die Namen von notorischen Ketzern wie Schreckenfuchs, Erasmus Reinhold und Sebastian Münster, die in Barozzis Forschungsüberblick genannt werden, unbeanstandet stehen. Zum anderen zeigt sich bereits in der zweiten, von Barozzi selbst durchgesehenen Auflage, dass die Menelaos-Schwärzung durchaus nicht die Tat eines einzelnen verwirrten Zensors war, sondern als systematisch durchgeführt betrachtet werden kann, da nun im gedruckten Vorwort der Name Menelaos schlicht ausgelassen, das heißt, gar nicht mehr mitgedruckt wurde (Barozzi 1589). ${ }^{63}$ In der italienischen Übersetzung des Werkes, das erstmals auch ausdrücklich von der Inquisition geprüft wurde, fehlte dann nicht nur der alttestamentarische Priester, es fehlten auch auch die Protestanten Schreckenfuchs, Reinhold und Münster (Barozzi 1607). ${ }^{64}$

\section{Ergebnisse für die frühneuzeitliche Zensur- und Astronomiegeschichte}

Barozzi erwähnte in seinem Vorwort auch Nikolaus Kopernikus' Namen und seine heliozentrische Theorie. Doch dieser Name und diese Theorie wurden weder 1585, 1598 noch 1607 expurgiert. ${ }^{65}$ Kopernikus stand nämlich nicht auf der Liste bekannter Ketzer, wenngleich seine astronomische Theorie unter bestimmten Umständen nach 1616 als Häresie angesehen wurde. Angesichts des confessional profiling, mit dem die katholischen Zensoren die publizistischen Leistungen protestantischer Autoren und Verleger durchkämmten, lässt sich durchaus von einer Zensur nach dem Prinzip ad hominem sprechen, einer Zensur, die sich nicht vorrangig auf Theorien bezog, sondern auf biographische Aspekte der Autoren (Sander \& Casalini 2017: 27-28). Diese Doktrin darf jedoch nicht vorschnell als Pauschalzensur bewertet werden, denn bei genauerer Betrachtung ergibt sich ein deutlich differenzierteres Bild, das die historische Zensurfor- 
schung in vielen Zusammenhängen bereits herausgearbeitet hat: Die Werke der Protestanten, so lange sie nicht theologischen Inhaltes waren, durften nämlich sehr wohl von Katholiken gelesen werden. Lediglich marginale Änderungen, wenn überhaupt, mussten die Bände erdulden. Gleichzeitig stammte der Sphaera-Traktat, der am häufigsten in den katholischen Indices genannt war, ausgerechnet aus der Feder eines Katholiken: Mauro da Firenze. Diese Zensur war hingegen klar inhaltlich, nicht biographisch motiviert, vertrat Mauro ja in seinen Annotationi durchaus streitbare theologische Positionen. Und zudem spiegelt die diskontinuierliche und heterogene Liste zensierter Sphaera-Traktate auch wider, dass die katholische Zensur keineswegs monolithisch und unwidersprüchlich arbeitete, sondern sich zwischen den einzelnen Ausgaben des Indexes durchaus Spannungen beobachten lassen. ${ }^{66}$

Diese Ergebnisse informieren in erster Linie über Praxis und Rationalität der Zensur. Darüber hinaus lassen sich jedoch auch Erkenntnisse über Dynamiken der Sphaera-Tradition festhalten. Das Repertoire insbesondere der in Frankfurt und Wittenberg gedruckten Sphaera-Bände, die in den italienischen, aber vor allem in den iberischen Indices auftauchen, gibt beispielsweise Aufschluss darüber, wie weit der Arm der Zensoren reichte, beziehungsweise wie die protestantischen Druckwerke auch überregional in erzkatholischen Gebieten bezogen werden konnten (Godman 2000: 168). Dieses Netzwerk der Verbreitung einzelner Ausgaben spiegelt sich auch in der Verbreitung der Inhalte dieser Ausgaben wider. Wenn etwa die Wittenberger Edition von 1531 bereits ein Jahr später in Venedig nachgedruckt wurde, bezeugt dies Dynamiken des Buchmarktes, die nötig waren, um erfolgversprechende Editionen wieder und wieder an unterschiedlichen Orten zu verlegen. Während die Karte dieser publizistischen Landschaft der Sphaera-Tradition noch zu zeichnen ist, vermittelt die Praxis der Zensoren durchaus eine Vorstellung ihrer Konturen.

Aus dieser Perspektive stellt sich zudem die Frage, inwieweit die Zensur Produktion und Rezeption der Sphaera-Werke beeinflusst hat. Deutlich ist jedenfalls, dass die Zensur die Lektüre der Traktate protestantischer Werke nur im Ausnahmefall für Katholiken unmöglich gemacht haben dürfte. Erhaltene Expurgationen zeigen zudem, dass kaum von einer Verstümmelung der Bände zu sprechen ist, sondern höchsten von minimalen, präzisen Eingriffen. Das vermeintlich deutlichste Opfer der Zensur, Melanchthons Vorwort, wurde zwar oft entfernt, aber die Ausgaben, die den Namen des Reformators gar nicht erst nannten, blieben hiervon verschont.

Die These, dass die Zensur astrologischer Inhalte in den Sphaera-Werken großen Einfluss auf die Astrologie als Ganzes gehabt hätte, lässt sich jedoch allein am Beispiel von Melanchthons Vorwort schwer belegen. Melanchthons Vorwort konnte, der Sache nach nicht im Geringsten verändert, 
dann problemlos gedruckt werden, wenn sein Name ausgelassen wurde. So klingen auch in anderen, ausdrücklich von der Inquisition untersuchten und genehmigten Werken durchaus apologetische Töne in Bezug auf die Astrologie an, etwa in Vorworten und Einführungen zu Ginés Rocamora y Torranos $(† 1612)$ Sphera del universo (1599) oder Luis de Mirandas Exposicion de la Esfera (1629) (Rocamora y Torrano 1599: 6v-12v; Giuntini et al. 1629: prol.). ${ }^{67}$ Zwar geht es in diesen Texten nicht ausdrücklich um juridische Aspekte der Astrologie, doch ist ein solcher Schwerpunkt auch in den zensierten oder expurgierten Stellen, die die Astrologie betreffen, nicht immer deutlich erkennbar: Weder Proklos' Traktat über Sonnen- und Mondfinsternisse, Sacroboscos Erwähnung der dies aegyptiaci in seinem Computus, noch Ausführungen zu den Kometen in Pedro de la Hera y de la Varras Reportorio können als Manifeste der divinatorischen Künste gelten. Die Zensur dieser Themen lässt sich in den Sphaera-Traktaten somit zwar punktuell finden, systematisch auf diese Themen hin untersucht und expurgiert wurden sie jedoch nicht. Das wiederum bedeutet nicht, dass die schwindende Relevanz dieser Themen nicht auch durch die Praxis der Inquisition bedingt wurde, aber die Zensur innerhalb der Sphaera-Tradition bestätigt einen solchen Befund eben nur bedingt (Hirai \& Vermij 2017). Vielmehr scheint eine vorläufige Analyse des Corpus der zahlreichen Sphaera-Traktate nahezulegen, dass die Thematik in diesem Publikationskontext aus noch zu eruierenden Gründen ohnehin eher an Boden verlor.

Evaluiert man diese Ergebnisse vor dem Hintergrund der in der Einleitung aufgegriffenen Gegenüberstellung der Thesen Woottons und Lüthys, lässt sich beiden Ansichten etwas abgewinnen. Die konzeptuellen und methodischen Entwicklungen, die sich auch in den Sphaera-Traktaten abzeichnen, sind von der Zensur, und damit vom Einfluss der Religion, letztlich nicht direkt betroffen. Die zensierten Inhalte beziehen sich also nicht auf die Aspekte der Astronomie, die für die Entstehung der modernen Wissenschaften als maßgeblich betrachtet werden. Insofern ließe sich sagen, die Zensur hatte kaum Einfluss auf die Generierung des Wissens in der Sphaera-Tradition. Diese Einsicht ist jedoch auch in Lüthys Sinne zu interpretieren: Der publizistische Erfolg der Sphaera-Traktate war möglicherweise genau der Tatsache zu verdanken, dass die Inhalte dieser Schriften in konfessioneller Hinsicht unbedenklich waren. Natürlich wäre es anachronistisch, diesen Aspekt dem Autor Johannes de Sacrobosco anzurechnen. Doch die vielen Editoren und Kommentatoren des 16. und 17. Jahrhunderts hatten in diesem mittelalterlichen Werk offenbar eine Grundlage gefunden, aktualisiertes kosmologisches und astronomisches Wissen zu publizieren, das dem entsprechen mochte, was Lüthy als "aseptic science and the culture of observational and experimental facts“ 
versteht. Gerade die vielen Tabellen und instrumentenbezogenen Inhalte der Sphaera-Traktate sprachen genau diese überkonfessionelle Sprache, in der sie von Autoren beziehungsweise Lesern über die Grenzen der Konfessionen hinweg verfasst beziehungsweise gelesen wurden. ${ }^{68}$

Funding Open access funding provided by Max Planck Society. 


\section{Anhang}

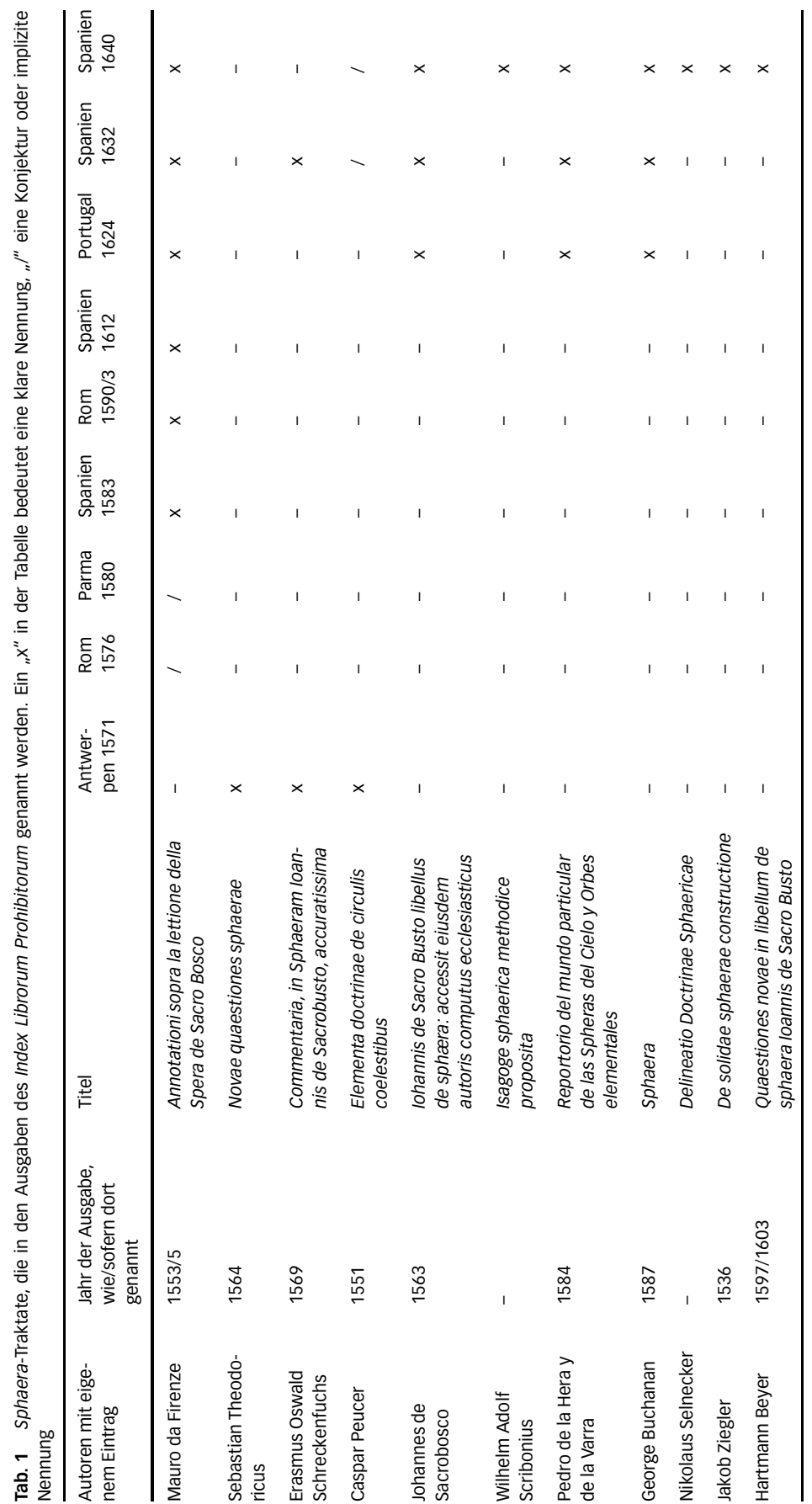




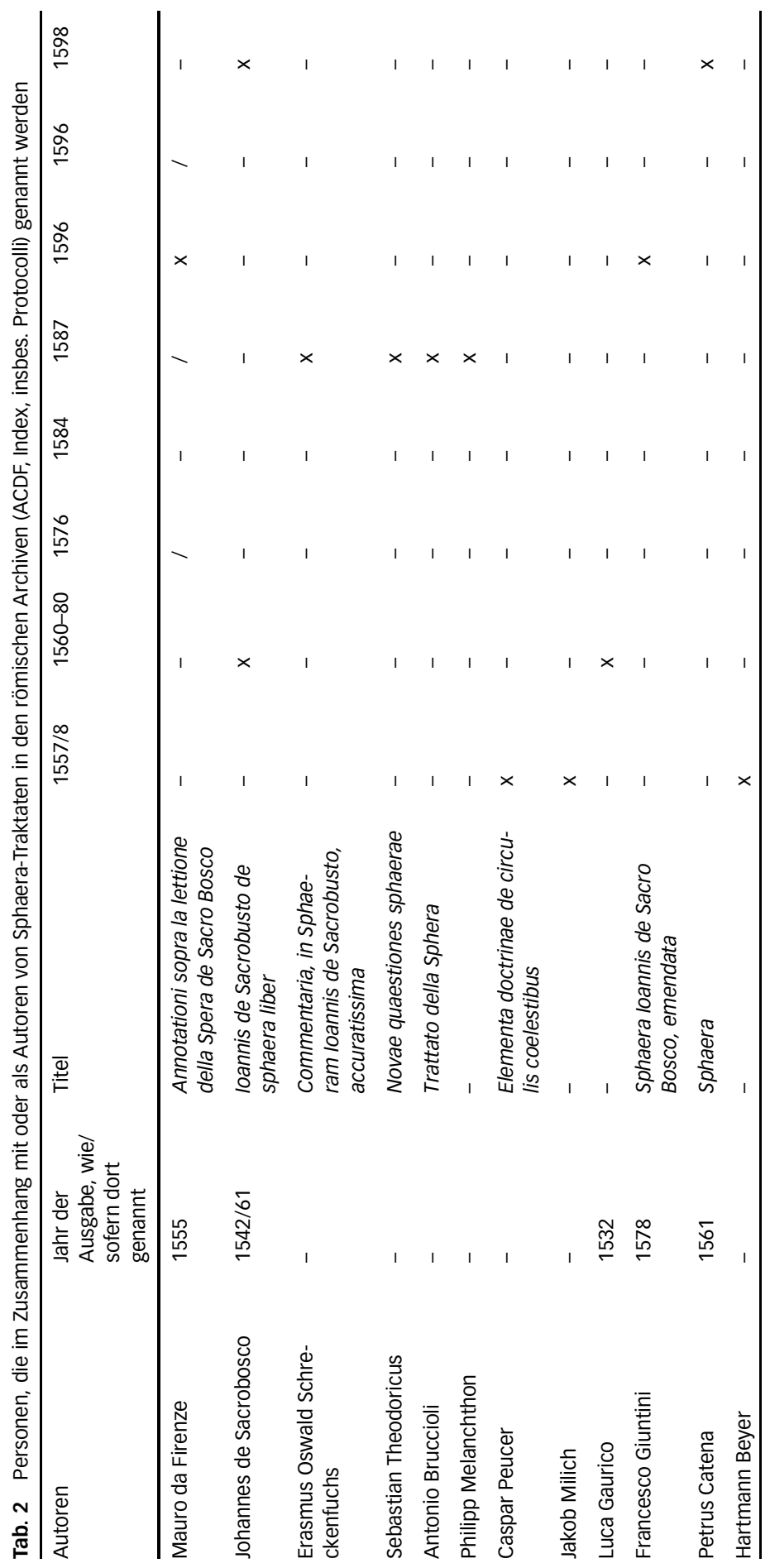




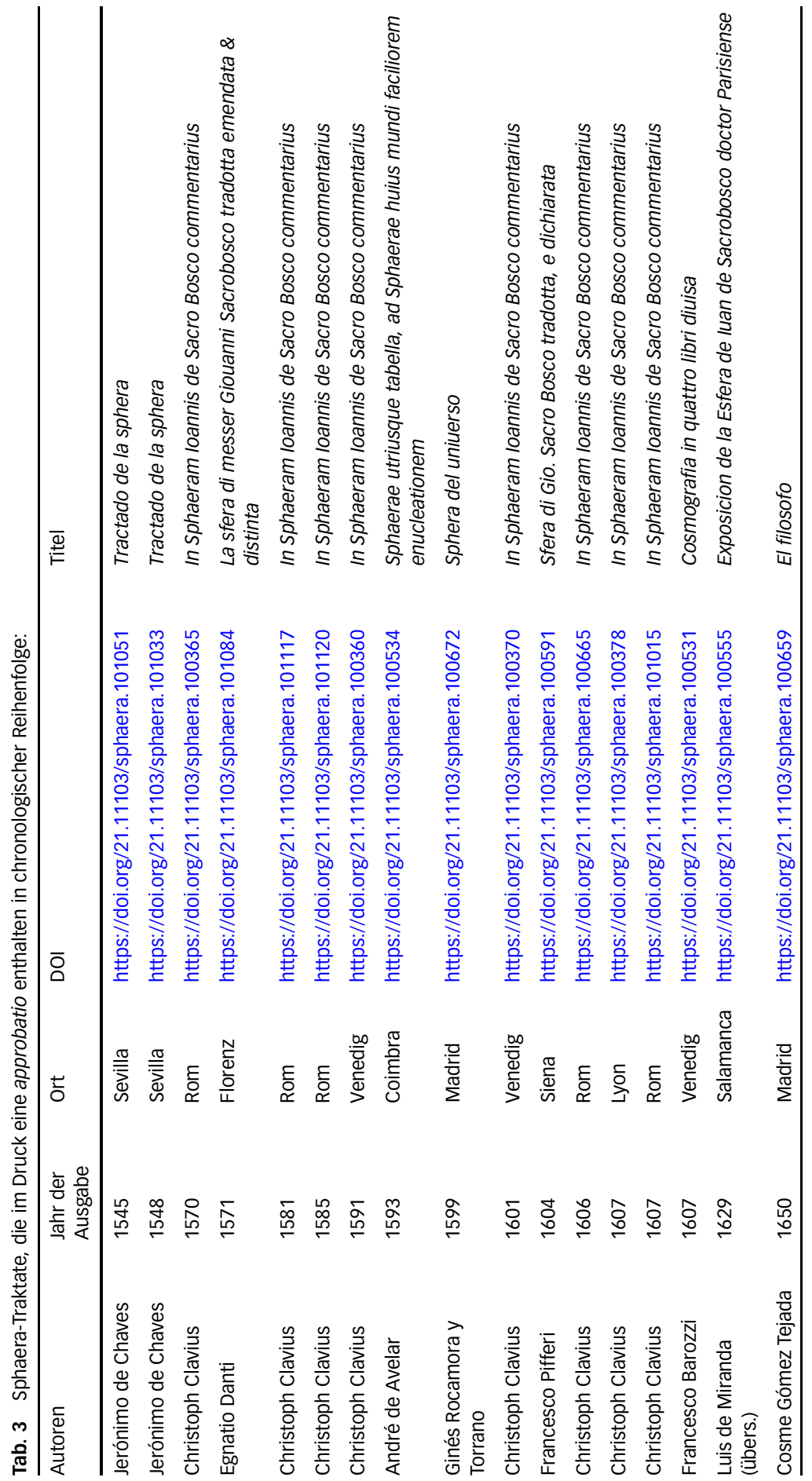


Open Access Dieser Artikel wird unter der Creative Commons Namensnennung 4.0 International Lizenz (http://creativecommons.org/licenses/by/4.0/deed.de) veröffentlicht, welche die Nutzung, Vervielfältigung, Bearbeitung, Verbreitung und Wiedergabe in jeglichem Medium und Format erlaubt, sofern Sie den/die ursprünglichen Autor(en) und die Quelle ordnungsgemäß nennen, einen Link zur Creative Commons Lizenz beifügen und angeben, ob Änderungen vorgenommen wurden.

\section{Anmerkungen}

1 Die Forschung für diesen Aufsatz wurde im Rahmen des Projektes „The Sphere. Knowledge System Evolution and the Shared Scientific Identity of Europe" am Max-PlanckInstitut für Wissenschaftsgeschichte durchgeführt. Ich möchte mich bei Leen Spruit und Matteo Valleriani für ihre Anmerkungen zu einer früheren Version dieses Aufsatzes bedanken. Zudem bedanke ich mich für die hilfreichen Kommentare der anonymen Gutachter. Claudia Burghof danke ich für die sprachliche Korrektur des Textes, Victoria Beyer für Korrekturen im englischen Abstract.

Folgende Abkürzungen werden in diesem Aufsatz benutzt:

ILI = (Bujanda u. a. 1984-2002).

CCMS $=$ (Baldini \& Spruit 2009)

Bände werden mit römischen Zahlen angegeben.

Sämtliche DOI-Links verweisen auf http://db.sphaera.mpiwg-berlin.mpg.de/ resource/Start [26.09.2018]. Dort können vollständige bibliographische Angaben eingesehen werden. $\mathrm{Zu}$ einer jüngeren historiographischen Kritik an Woottons These, dass der Aristotelismus in der Wissenschaftlichen Revolution eine marginale Rolle spielte, vgl. (Sgarbi 2017).

2 Vgl. neben CCMS auch beispielsweise in Pardo Tomás (1991); Dinis (2005); Ariew (2013); Bertoloni Meli (1988); Brevaglieri (2009); Gorman (1996); Hellyer (1996); Sander (2017); Ricci (2008); Beretta (2005). Die meisten dieser Beispiele konzentrieren sich auf die Zensur der Werke eines bestimmten, meist berühmten Autors. Insbesondere Ugo Baldini hat sich um eine Aufarbeitung der philosophischen oder wissenschaftlichen Zensur in zahlreichen Studien bemüht. (Vgl. insbes. Baldini 1985 und Baldini 2001.) Viele weitere, kleinere Studien wären zu erwähnen. Doch gerade die spanische Zensur wird deutlich weniger mit Blick auf ihr Wirken in wissenschaftlichen oder philosophischen Zusammenhängen berücksichtigt.

3 Vgl. auch Wootton (2015: 228).

$4 \quad$ Vgl. als bekannte Ausnahme die Zensur von Biancani (1620). Hierzu siehe etwa auch in Ceglia (2003).

5 Zu den Abläufen der Zensur, vgl. insbes. auch Mayer (2013); Zedelmaier (2015: 75-88); Hasecker (2017).

6 Hierzu ausführlich in ILI. Zur frühen Buchzensur, vgl. auch Minnich (2010); Thomsett (2010); Monfasani (1988).

7 Gleichzeitig, und hiervon vielfach kaum zu unterscheiden, diente die Zensur aber auch der Sicherung politischer Macht und Ansprüche sowie der Wahrung traditioneller Werte und guter Sitten.

8 Die italienische Zensur ist in Bezug auf ihre Abläufe und ihre Auswirkung deutlich besser erforscht, vgl. neben der bereits angeführten Literatur auch Paolin \& Del Col (1991); Del Col \& Paolin (2000); Prosperi (2003); Rebellato (2008); Prosperi et al. (2010); Del Col (2012; Mayer 2015).

9 Hiervon zu unterscheiden sind allerdings sogenannte privilegia, die die Urheber-oder marktwirtschaftlichen Rechte von Verleger oder Autor schützen, nicht aber generell als religiöse oder sittliche Unbedenklichkeitsbescheinigung zu verstehen sind, vgl. dazu 
Castellani (1888); Peixoto (1966); Labarre (1970); Witcombe (1991); Armstrong (2002); Witcombe (2004); Bently et al. (2010); Squassina (2016); Barbier \& Ducreux (2005); Grendler (1977).

10 Es ist hier ebenfalls zu berücksichtigen, dass insbesondere in Italien auch Zensur auf Lokalebene, also nicht von Rom aus, betrieben wurde. Diese Indices werden in diesem Aufsatz nur im Fall von Mauro da Firenze mitberücksichtigt, da sie ansonsten für die Sphaera-Tradition irrelevant erscheinen.

11 Einzig bei der Erläuterung der Sonnenfinsternis kommt Sacrobosco auf einen direkt theologischen Zusammenhang zu sprechen. (Vgl. Thorndike 1949: 117.)

12 Eine direkte Überlieferung des Prozesses ist nicht erhalten. (Vgl. Anonym 1792: 15; Alessandrini 1955: 273; Thorndike 1949: 53-54.) Cecco wurde als relapsus verurteilt, also als jemand, der hartnäckig und gegen bessere Belehrung wiederholt an einer Ketzerei festgehalten hat.

13 Vgl. https://doi.org/21.11103/sphaera.100273,https://doi.org/21.11103/sphaera.100021, https://doi.org/21.11103/sphaera.101057, https://doi.org/21.11103/sphaera.100047.

14 Vgl. https://doi.org/21.11103/sphaera.100822.

15 Vgl. BAV, Vat. Lat. 6207, fols. 220r-239v: Anonymous, Instructiones nonnulle circa libros nominatim prohibitos in S.to Indice (Rom, 1557-1558).

16 Vgl. https://doi.org/21.11103/sphaera.100251.

17 Vgl. https://doi.org/21.11103/sphaera.101034.

18 Vgl. https://doi.org/21.11103/sphaera.100138.

19 Der Text bei Peucer lautet: „Cumque doctrinam Ecclesiae incorruptam et studiose legas." Im Index wird hingegen „Cumque doctrinam Ecclesiae corruptam incorruptam et studiose legas“ zitiert. Der Passus selbst scheint eher unbedenklich.

20 Vgl. https://doi.org/21.11103/sphaera.101080.

21 Vgl. https://doi.org/21.11103/sphaera.101072.

22 Siehe zu Beginn von (Montana 1571): „Qui vero de religione non tractant, a theologis catholicis jussu episcoporum et inquisitorum examinati et approbati, permittuntur."

23 Das Instrument der Expurgation wurde schon im Tridentiner Index 1564 eingeführt. Der Index selber enthält jedoch keine Expurgationsanweisungen.

24 Vgl. im Index des „Magister Sacri Palatii“ (ILI X, 832) von 1576, in Giovanni Deis Index Authorum von 1576 (ILI I, 291), im Index aus Parma (ILI IX, 115) von 1580, aus Turin um 1580 (ILI IX, 761), aus Neapel von 1583 (IX, 773) sowie in einer anonymen Liste aus dem ACDF (CCMS I, 320), die zwischen 1584 und 1587 entstanden ist.

25 Nur in ILI X, 832 wird die Schrift auf 1553 datiert. Laut (Inghirami 1844: 354) wurde das Werk auch 1557 gedruckt, allerdings ebenfalls in Florenz. Eine Kopie ist nicht erhalten.

26 Vgl. https://doi.org/21.11103/sphaera.101035. Alle Zahlen in Klammern sind Seitenangaben zu Mauro da Firenze \& Sacrobosco 1550.

27 Vgl. https://doi.org/21.11103/sphaera.101009. Dieses Werk ist eher mathematisch-kosmographisch ausgerichtet.

28 Am Ende des Bandes Mauro da Firenze \& Sacrobosco (1550) werden weitere Schriften aufgezählt, die Mauro verfasst haben soll. Sie wurden nicht gedruckt, sondern sind, wenn überhaupt, handschriftlich erhalten. Auf eine von ihm verfasste Meteora verweist Mauro im Text (30).

29 Dieses Horoskop wird erklärt in Mauro da Firenze \& Sacrobosco (1550: 205-213).

30 Alle Seitenangaben nach (Mauro da Firenze \& Sacrobosco 1550). Weitere erwähnte Autoren beziehungsweise Autorengruppen: Thebit: 27; Alfragano: 27; Peuerbach: 42; Ptolemäus: 42; Peter Apian: 46; Vitruv: 58; Dante Alighieri: 161; Boethius: 166; Francesco Petrarca: 161; Häretiker: 167, 188; Athanasius der Große: 169; Epikureer: 191; Richard von St. Viktor: 174; Thomas von Aquin: 176, 187, 188; Scotisten: 176; Alfonso de Castro: 178; Gregor der Große: 185; Cicero: 190; Macrobius: 190; Johannes Chrysostomos: 195; Homer: 195; Laktanz: 195. 
31 Piccolominis Name wurde aus dem Text entfernt und verblieb nur in den Marginalien. Namentlich nicht genannte „Häretiker“ erwähnt Mauro ebenfalls $(167,188)$. Zu Johannes Bernhardi, vgl. Anm. 34.

32 Zur Bibel, (NT), vgl. auf S. 147, 149, 163, 164, 164, 165, 166, 171, 179, 183, 184, 185, 185, 187, 191, 197, 198; (AT): 163, 174, 175, 183, 191; siehe auch 94, 95, 147, 151.

33 Im Anhang fügt Mauro wesentliche Textergänzungen nach Drucklegung hinzu.

34. In Bezug auf die Unsterblichkeit vertritt Mauro eine anti-naturalistische Position, das heißt, ein rein philosophischer Beweises ist unmöglich zu erbringen. Dies hatte die Bulle Apostolici regiminis (1513) ausdrücklich untersagt. Mauro argumentiert gegen die Position von Velcurio (1537). Vgl. zur Bulle insbesondere Constant (2002). Mauros Position zur Willensfreiheit ist komplexer. Einige Bemerkungen finden sich etwa in Mauro da Firenze \& Sacrobosco (1550: 296).

35 Vgl. dazu die Handschrift in Madrid, Biblioteca Nacional de España, Mss/9273. Vgl. Hurtado Torres (1982: 52). Zu Luis Ramón de Aragón y Fernández de Córdoba, vgl. (Gassó 2004).

$36 \mathrm{Zu}$ bedenken ist hierbei aber, dass die erste tridentinische Regel des Indexes weiterhin besagte, dass alle einst verurteilten Werke und Autoren dies auch weiterhin bleiben.

37 Vgl. CCMS I, 256: „In Sphera, Erasmi Osvaldi [Schreckenfuchsii], Antonij Bruccioli [https://doi.org/21.11103/sphaera.101028], Melancthonis [...] Gasparis Peuceri elementa doctrinae de circulis coelestibus à $\mathrm{p}$ (rim)o motu et eiusdem liber de dimentione terrae."

38 Vgl. auch Martins (2011); Manning (2009: 24); Pardo Tomás (1991). In dem Index expurgatorius von Juan de Mariana (1584) hingegen fehlen sämtliche Sphaera-Traktate.

39 Gessner et al. (1583: 492): „Ioannis de Sacrobusto liber de sphaera, impressus Wittenbergae, et olim Venetiis, cum additionibus quibusdam. Eiusdem autoris computus, anni et mensium descriptionem continens, simul excusa in Germania, cum praefatione Philippi Melancht." (Vgl. Sandoval y Rojas 1612: 174; Mascarenhas 1624: 427; Zapata 1632: 197.)

40 Vgl. Mascarenhas (1624: 147, 820): „Eius Sphaera, cum Commentariis quorundam, in editione Vvitenbergensi, anno 1563 [https://doi.org/21.11103/sphaera.101070], Corrigenda. In libri fronte, dele, cum Praefatione Philoppi Melanthonis. - Et in medio totius voluminis, (nec enim folia numerantur) in titulo, Libellus Ioannis de Sacrobosco, de anni ratione, etc. dele rursus eadem verba; cum Praefatione etc. Ipsam quoque Praefationem, quae incipit, Ingens $\mathcal{E}$ manifesta etc. cum suo titulo. Quia vero cum praedicta Sphaera, et cum Compendio Pierij Valeriani, circumfertur Tractatus de Eclypsibus, ex Proclo Lypsio, is totus expungendus est. Circumfertur praeterea in quibusdam editionibus, post praedictam Sphaeram, Computus Ecclesiasticus, in quo, sub titulo, Kalendas, Nonas, $\mathcal{E}$ Idus, deleatur totus paragraphus. Notandum etiam, quod in quolibet mense etc. - Tum praeterea Annotationes quaedam Marci Mauri in Sacrobosci Sphaeram expungi debent."

41 Beide Traktate wurden ab 1551 oft mit in Sphaera-Bände eingefügt, allerdings nie in Wittenberg. Ob diese „synoptische“ Kontrolle gängig war, bleibt ungewiss, da hierfür andere Fälle zu untersuchen wären.

42 Vgl. https://doi.org/21.11103/sphaera.100460.

43 Beispielsweise wurde ein Kapitel zur „Bedeutung der Kometen“ in Hera y de la Varra (1584: 63v) beanstandet.

44 Zu einigen dieser Autoren, vgl. Brosseder (2004). Zu Beyer, vgl. Anm. 17. Im Index werden jedoch zwei Ausgaben (Venedig 1597, Frankfurt 1603) genannt, die unbekannt sind. Der letzte bekannte Druck datiert von 1573 (https://doi.org/21.11103/sphaera.100298). Zu den übrigen Autoren, vgl. Scribonius (1581); Ziegler (1536); Selnecker (1577).

$45 \mathrm{Zu}$ einer Zensur von Biancani (1620), vgl. die Handschrift in Rom, Archivum Romanum Societatis Iesu, FG 655, ff. 156r-160v. (Vgl. auch Baldini 1992: 111, Anm. 73 und Anm. 4.)

46 Vgl. auch Biasiori (2010); Baldini (1985). 
47 Die Ausgaben stammen aus Rom, Venedig und Lyon. Das initiale Approbationsschreiben (https://doi.org/21.11103/sphaera.100365) stammt von Papst Pius V. beziehungsweise enthält die Angabe „cum licentia superiorum“. Ausgaben ab 1606 wurden zudem vom "Magister Sacri Palatii“ durchgesehen. In folgenden Ausgaben findet sich keine Approbation: https://doi.org/21.11103/sphaera.100367 (1596, Venedig), https://doi.org/21.11103/sphaera.100673 (1601, Venedig), https://doi.org/21.11103/ sphaera.100368 (1602, Lyon), https://doi.org/21.11103/sphaera.100369 (1602; Saint Gervais), https://doi.org/21.11103/sphaera.100377 (1607; Saint Gervais), https://doi. org/21.11103/sphaera.100380 (1607; Genf), https://doi.org/21.11103/sphaera.100381 (1608; Saint Gervais), https://doi.org/21.11103/sphaera.100369 (1602; Saint Gervais), https://doi.org/21.11103/sphaera.100383 (1611; Mainz), https://doi.org/21.11103/ sphaera.100382 (1618; Lyon). Für eine Übersicht über alle 22 bekannten Editionen, vgl. https://doi.org/21.11103/sphaera.100732.

48 Vgl. https://doi.org/21.11103/sphaera.100377.

49 Vgl. https://doi.org/21.11103/sphaera.100672,https://doi.org/21.11103/sphaera.100555. Vgl. auch https://doi.org/21.11103/sphaera.100659.

50 Vgl. CCMS III, 2610: „Sphera Io. de sacro bosco cum ephemeride Lucae Gaurici pro anno 1532." Dieser Band lässt sich nicht identifizieren.

51 Vgl. CCMS III, 2692: „Annotationi sopra la lettione della sphera del sacrobosco et cet. autore m. mauro fiorentino [...]. Sphaera Ioannis de Sacro Boscho emendata a Fr. Iunctino Theologo Fiorentino“. https://doi.org/21.11103/sphaera.100311. (CCMS IV, 3085, verzeichnet, dass eine Lesegenehmigung ausgestellt wurde, allerdings nicht für Mauros Annotationi, sondern für dessen italienische Sphaera-Übersetzung von 1537).

52 Vgl. CCMS III, 2723: „Ioannis de Sacrobusto sphera ven (etijs) apud frane. Rampazitum 1561. [https://doi.org/21.11103/sphaera.100162]“; „Item sphera Petri Cathena. Patavij apud Gratiosum porcaccinum 1561 [https://doi.org/21.11103/sphaera.101105]“; „Ioannis de Sacrobusco Sphera Ven. Item Vitemberge apud Io. Lufft 1542 [nicht bekannt]“; „Ioannis de Sacrobosco de anni ratione, seu computus ecclesiasticus cum praefat (ion)e Melanctonis Lut (et)ae Gul. Cavel. 1561 [https://doi.org/21.11103/sphaera.100211]“.

53 Das Verbot betraf mit einer Ausnahme pauschal alle seine Werke.

54 Vgl. https://doi.org/21.11103/sphaera.100118. Zu Sessa, vgl. Curi Nicolardi (1984); Svoljšak \& Kocjan (2016); Vianello (1970).

55 Vgl. beispielsweise den Nachdruck von Euklid et al. (1536) in Euklid et al. (1539). Vgl. auch die venezianischen Drucke bei Sessa oder da Sabbio mit Beteiligung Melanchthons: Alciati et al. (1532); Dubois et al. (1537); Vergil et al. (1539).

56 Vgl. ILI IV, 1000: „Philippus Melanchthon, livros nos quaes estão suas annotaçoes que não fallão em cousa de religião christãa. Corrão apagado ho nome do author, et nao venha mais o reino. Epistolas cortense."

57 Vgl. https://doi.org/21.11103/sphaera.100137: München, Bayerische Staatsbibliothek, Astr. u. 161, Titelseite, Brieftitel unversehrt [Besitzvermerk Münchener Jesuitenkolleg 1581]. https://doi.org/21.11103/sphaera.101029: Augsburg, Staats- und Stadtbibliothek, Math 745, Titelseite, Brieftitel. https://doi.org/21.11103/sphaera.100180: Emden, Johannes a Lasco Bibliothek, Philos. $8^{\circ} 1320$ M, Titelseite, Brieftitel. https://doi.org/21.11103/ sphaera.100211: Paris, Bibliothèque nationale de France, Microfilm FRBNF37254729, Brieftitel. https://doi.org/21.11103/sphaera.100118: London, British Library, 717.d.54, Titelseite, Briefende.

58 Vgl. https://doi.org/21.11103/sphaera.100137. Siehe vorangehende Fußnote.

59 Oosterhof vermutet dahinter eine protestantische Hand aufgrund Pierres Rolle im Konstanzer Konzil.

60 Vgl. https://doi.org/21.11103/sphaera.100210: Rom, Nationale Zentralbibliothek, 201. 37.A.37, fol. 51r [Das Exemplar stammt aus dem Besitz der Jesuiten in Rom]; https:// doi.org/21.11103/sphaera.101094: Rom, Bibliothek Alessandrina, A g.48 1, S. 81 [Besitzvermerk: Antonij Lanfredinj. Lanfredino war Florentiner. Als weiterer Vorbesitzer lässt 
sich Costantino Caetani bestimmten, ein römischer Kleriker, dessen Bibliothek 1666 an die Alessandrina überging].

61 Vgl. https://doi.org/21.11103/sphaera.101111: Lyon, Bibliothèque municipale, B 509795, Titelseite. Vgl. auch Anm. 51 und 67. Der Fall von Giuntini, der auch einen SphaeraKommentar verfasste, der in den analysierten Indices jedoch nicht eigens aufgeführt wird, ist komplizierter. Seine Auseinandersetzung mit der Inquisition verdankt sich keineswegs nur seinen astrologischen Werken. Vgl. CCMS II, 1878. Die Ausgabe von Giuntinis Speculum in dem Exemplar in Sevilla, Biblioteca Rector Machado y Núñez, A Res.03/3/14, wurde beispielsweise sehr umfangreich expurgiert.

62 Vgl. https://doi.org/21.11103/sphaera.100518: Durham, Duke University Libraries, David M. Rubenstein Rare Book and Manuscript Library, QB41 .B2 1585 c.1, fol. B5v.

63 Vgl. https://doi.org/21.11103/sphaera.100517.

64 Vgl. https://doi.org/21.11103/sphaera.100531.

65 Hierzu muss jedoch gesagt werden, dass Barrozzi der kopernikanischen Theorie nicht anhing, sondern diese vielmehr kritisierte.

66 Vgl. Godman (2000: 21): „Often and rightly described as a tribunal, the Roman Inquisition was more. It was also a forum for debate."

67 Vgl. https://doi.org/21.11103/sphaera.100672; https://doi.org/21.11103/sphaera.100555. Luis de Miranda paraphrasiert Giuntinis Anmerkungen zur Sphaera, darunter auch dessen Astrologieapologetik. Vgl. auch in Anm. 51.

68 Der Punkt, der aus Sicht der Zensur am Kopernikanismus heterodox ist, betraf die Annahme einer heliozentrischen Kosmologie als physikalische Theorie, nicht nur als mathematische Hypothese. Vgl. hierzu Clarke (2011); Wootton(2015: 380-399). Für die in den Sphaera-Traktaten gelieferten Tabellen und instrumentenbezogenen Inhalte, aber auch für viele andere Inhalte war die physikalische Theorie letztlich unerheblich, da für die Benutzung der Instrumente, beispielsweise eines Astrolabiums, die geozentrische Kosmologie als benchmark fungierte und nur unter dieser Voraussetzung überhaupt (ohne tiefgreifende Modifikationen) benutzt werden konnte. Vgl. dazu Valleriani (2017: 435, 438): „The instruments used to calculate the time and one's position on the Earth's surface had been used since antiquity and during the later epochs on the basis of the geocentric spherical system described in the treatise. [...] Astrolabes, for instance, were instruments conceived back in antiquity on the basis of a geocentric worldview. The advent of the Copernican doctrine did not change the habit or the reasons for using such instruments. Their functioning was based on a precise stereometric projection of the eighth sphere - the sphere of the fixed stars - onto the plate of the instrument. The use of the astrolabe therefore always presupposes the knowledge of the geocentric structure of the world“. Dieser Aspekt kann an dieser Stelle nicht weiter vertieft werden, da er ein anderes Thema betrifft.

\section{Literatur}

Alciati, Andrea, Philipp Melanchthon und Guillaume Budé 1532. And. Alciati Libellus de ponderibus et mensuris. Item Budaei quaedam de eadem re, adhuc non uisa. Item Philippi Melanchthonis, de ijsdem ad Germano[rum] usum, sententia. Alciati quoq[ue] et Philippi Mela[n]chthonis in laudem iuris ciuilis orationes du[a]e elegantissim[a]e. Venedig: M. Sessa.

Alessandrini, Mario 1955. Cecco D’Ascoli. Rom: Casini.

Anonym 1792. Nascita, vita, processo, e morte di F. degli Stabili volgarmente detto lecco d'Ascoli quale per $i$ suoi errori fu condannato ad effergli tagliate le vene della fronte, e gettato alle fiamme: Col suo ritratto in rame, come si vedo qui annesso. Florenz: G. Ponziani. 
Ariew, Roger 2013. Censorship, Condemnations, and the Spread of Cartesianism. In: Mihnea Dobre und Tammy Nyden-Bullock (Hg.). Cartesian Empiricisms. Dordrecht: Springer: 25-46.

Armstrong, Elizabeth 2002. Before Copyright. The French Book-Privilege System, 1498-1526. Cambridge; New York; Port Chester: Cambridge University Press.

Arnold, Claus 2008. Die römische Zensur der Werke Cajetans und Contarinis (1558-1601): Grenzen der theologischen Konfessionalisierung. Paderborn: Schöningh.

Baldini, Ugo 1985. Una fonte poco utilizzata per la storia intellettuale: le censurae librorum e opinionum nell'antica Compagnia di Gesù. Annali dell'Istituto storico italo-germanico in Trento (11):19-67.

Baldini, Ugo 1992. Legem impone subactis: studi su filosofia e scienza dei Gesuiti in Italia, 1540-1632. Rom: Bulzoni.

Baldini, Ugo 2001. The Roman Inquisition's Condemnation of Astrology: Antecedents, Reasons and Consequences. In: Gigliola Fragnito (Hg.). Church, Censorship, and Culture in Early Modern Italy. Cambridge; New York: Cambridge University Press: 79-110.

Baldini, Ugo und Leen Spruit (Hg.) 2009. Catholic Church and Modern Science: Documents from the Archives of the Roman Congregations of the Holy Office and the Index. Bd. 1: Sixteenth-century Documents. Rom: Libreria Ed. Vaticana.

Barbier, Frédéric und Marie-Elizabeth Ducreux (Hg.) 2005. Libri prohibiti: la censure dans l'espace habsbourgeois 1650-1850. Leipzig: Leipziger Univ.-Verl.

Barbieri, Edoardo 2011. Giovanni della Casa e il primo processo veneziano contro Antonio Brucioli. In: Stefano Carrai (Hg.). Giovanni Della Casa, ecclesiastico e scrittore: atti del convegno, Firenze-Borgo San Lorenzo, 20-22 novembre 2003. Rom: Edizioni di storia e letteratura: 31-69.

Barozzi, Francesco 1585. Cosmographia in 4 libros distributa. Venedig: G. Perchacino.

Barozzi, Francesco 1589. Cosmographia in 4 libros distributa. Venedig: G. Perchacino.

Barozzi, Francesco 1607. Cosmografia in quattro libri divisa: la quale con sommo ordine, $e$ marauigliosa facilta, e breuita introduce alla grande mathematica costruzione di Tolomeo, E a tutta l'astrologia. Venedig: G. Perchacino.

Bennett, Jim und Domenico Bertoloni Meli (Hg.) 1994. Sphaera mundi: Astronomy Books in the Whipple Museum 1478-1600. Cambridge: Whipple Museum of the History of Science.

Bently, Lionel, Martin Kretschmer und Ronan Deazley (Hg.) 2010. Privilege and Property: Essays on the History of Copyright. Open Book Publishers. URL: http://books. openedition.org/obp/1046 (08.10.2017).

Beretta, Francesco 2005. Orthodoxie philosophique et Inquisition romaine aux 16e-17e siècles. Historia philosophica (3):67-96.

Bertoloni Meli, Domenico 1988. Leibniz on the Censorship of the Copernican System. Studia Leibnitiana 20(1): 19-42.

Biagioli, Mario 2002. From Book Censorship to Academic Peer Review. Emergences: Journal for the Study of Media E Composite Cultures 12(1): 11-45.

Biancani, Giuseppe 1620. Sphaera mundi, seu, Cosmographia: demonstratiua, ac facili methodo tradita: in qua totius mundi fabrica, vna cum nouis, Tychonis, Kepleri, Galilaei, aliorumq[ue] astronomorum adinuentis continetur: accessere I., Breuis introductio ad geographiam, II., Apparatus ad mathematicarum studium, III., Echometria, idest geometrica traditio de echo. Bologna: S. Bonomius.

Biasiori, Lucio 2010. Il controllo interno della produzione libraria nella Compagnia di Gesù e la formazione del Collegio dei Revisori generali (1550-1650). Annali della Scuola Normale Superiore di Pisa, Classe di Lettere e Filosofia 2(1): 223-249.

Brevaglieri, Sabina 2009. Science, Books and Censorship in the Academy of the Lincei: Johannes Faber as Cultural Mediator. In: Maria Pia Donato und Jill Kraye (Hg.). Conflicting Duties: Science, Medicine, and Religion in Rome, 1550-1750. London; Savigliano: Warburg Institute; Nino Aragno Editore: 109-133.

Brosseder, Claudia 2004. Im Bann der Sterne: Caspar Peucer, Philipp Melanchthon und andere Wittenberger Astrologen. Berlin: Akademie Verlag.

Bruni, Flavia 2013. The Book Inventories of Servite Authors and the Survey of the Roman Congregation of the Index in Counter-Reformation Italy. In: Malcolm Walsby und Natasha Constantinidou (Hg.). Documenting the Early Modern Book World: Inventories 
and Catalogues in Manuscript and Print. Library of the Written Word. Leiden: Brill: 207-230.

Buchanan, George 1587. Sphaera. Herborn: Corvinus.

Bujanda, Jésus Martinez de, Francis M. Higman, James K. Farge, René Davignon, Ela Stanek, Léon- E. Halkin und Marcella Richter (Hg.) 1984-2002. Index des livres interdits. Québec/Genf: Ed. de l'Université de Sherbrooke/Éditions Médiaspaul et la Librairie Droz de Genève.

Caponetto, Salvatore 2000. Melantone e l'Italia. Turin: Claudiana.

Caravale, Giorgio 2011. Forbidden Prayer: Church Censorship and Devotional Literature in Renaissance Italy. Farnham, Surrey, England; Burlington, VT: Ashgate.

Caravale, Giorgio 2014. PANCIATICHI, Bartolomeo. In: Dizionario Biografico degli Italiani. http://www.treccani.it//enciclopedia/bartolomeo-panciatichi_(Dizionario-Biografico) (20.3.2018).

Castellani, Carlo 1888. I privilegi di stampa e la proprietà letteraria en Venezia dalla introduzione della stampa nella città fin verso la fine del secolo XVIII. Venedig: Fratelli Visentini.

Ceglia, Francesco Paolo de 2003. Additio illa non videtur edenda: Giuseppe Biancani, Reader of Galileo in an Unedited Censored Text. In: Mordechai Feingold (Hg.). The New Science and Jesuit Science: Seventeenth Century Perspectives. Dordrecht; Boston, MA: Kluwer Academic Publishers: 159-186.

Cirella, E. O. und A. Rinaldi 2015. The Manipulation of Images in Ancient Books: The Case of Sacrobosco's De Sphaera Mundi. In: András Holl, Soizick Lesteven, Dianne Dietrich und Antonella Gasperini (Hg.). Library and Information Services in Astronomy VII: Open Science at the Frontiers of Librarianship: Proceedings of a Conference Held at Astronomical Observatory of Capodimonte, Naples, Italy, 17-20 June 2014. San Francisco: Astronomical Society of the Pacific: $173-175$.

Clarke, Desmond M. 2011. Hypotheses. In: Desmond M. Clarke und Catherine Wilson (Hg.). The Oxford Handbook of Philosophy in Early Modern Europe. Oxford: Oxford University Press: 249-271.

Del Col, Andrea 1980. Il controllo della stampa a Venezia e i processi di Antonio Brucioli (1548-1559). Critica storica (17): 457-510.

Del Col, Andrea 2012. L'inquisizione in Italia: dal XII al XXI secolo. Milano: Mondadori.

Del Col, Andrea und Giovanna Paolin (Hg.) 2000. L'Inquisizione romana: metodologia delle fonti e storia istituzionale: atti del seminario internazionale, Montereale Valcellina, 23 e 24 settembre 1999. Triest; Montereale Valcellina (Pordenone): Università di Trieste; Circolo culturale Menocchio.

Constant, Eric A. 2002. A Reinterpretation of the Fifth Lateran Council Decree Apostolici regiminis (1513). The Sixteenth Century Journal (33/2): 353-379.

Crowther, Kathleen, Ashley Nicole McCray, Leila McNeill, Amy Rodgers und Blair Stein 2015. The Book Everybody Read: Vernacular Translations of Sacrobosco's Sphere in the Sixteenth Century. Journal for the History of Astronomy (46/1): 4-28.

Curi Nicolardi, Silvia 1984. Una società tipografico-editoriale a Venezia nel secolo XVI: Melchiorre Sessa e Pietro di Ravani (1516-1525). Florenz: Olschki.

Dinis, Alfredo 2005. Censorship and Freedom of Research among the Jesuits (XVIth-XVIIIth centuries): The Paradigmatic Case of Giovanni Battista Riccioli (1598-1671). In: Luís Miguel Carolino und Carlos Ziller Camenietzki (Hg.). Jesuitas, ensino e ciência: séc. XVI-XVIII. Casal de Cambra, Portugal: Caleidoscópio: 27-57.

Dowden, Ken 1999. Menelaos. In: Karel van der Toorn, Bob Becking und Pieter Willem van der Horst (Hg.). Dictionary of Deities and Demons in the Bible. Leiden; Boston: Brill: 565-566.

Dubois, François, Bartholomaeus Latomus, Philipp Melanchthon und Marcus Tullius Cicero 1537. In Reliquas M. T. Ciceronis Orationes F. Sylvii Ambiani, Philippi Melanchthonis, B. Latomi, E aliorum, Lucubrationum pars altera, quarum Catalogum proxima à prafatione pagina reperies.: Rerum ac verborvm in iisdem lucubrationibus memorabilium omnium diligentissimus. Index. Quaedeam multo quàm antea emendatius, quaedam nunc primum in lucem edita: id quod studiosi his usuri facile aestimabunt. Venedig: Giovanni Antonio Nicolini da Sabbio. 
Eisenstein, Elizabeth L. 2005. The Printing Revolution in Early Modern Europe. Cambridge; New York: Cambridge University Press.

Esteve, Cesc 2018. Disciplining History: Censorship, Theory and Historical Discourse in Early Modern Spain. New York: Routledge.

Euklid, Georg von Peuerbach, Johannes Vögelin und Philipp Melanchthon 1536. Elementa Geometriae. Ex Evclide singulari prudentia collecta a Ioanne Vogelin professore Mathematico in schola Viennensi. Arithmeticae Practica per Georgium Peurbachium Mathematicum. Cum prafacione Philippi Melanthonis. Wittenberg: J. Klug.

Euklid, Georg von Peuerbach, Johannes Vögelin und Philipp Melanchthon 1539. Elementa geometriae ex Evclide singulari prudentia collecta à Ioanne Vogelin ... Arithmeticae practica per Georgium Peurbachium ... Cum prafacione Philippi Melanthonis. Venedig: Giovanni Antonio Nicolini da Sabbio.

Finocchiaro, Maurice A. 2005. Retrying Galileo, 1633-1992. Berkeley: University of California Press.

Fragnito, Gigliola (Hg.) 2001. Church, Censorship, and Culture in Early Modern Italy. Cambridge; New York: Cambridge University Press.

Gassó, Joan Yeguas 2004. El testament de Luis II Fernández de Córdoba (+1642). Quaderns de El Pregoner d'Urgell (17): 49-82.

Gentile, Sebastiano und Carlos Gilly 2001. Marsilio Ficino e il ritorno di Ermete Trismegisto $=$ Marsilio Ficino and the Return of Hermes Trismegistus. Firenze: Centro Di.

Gessner, Conrad, Johann Jacob Fries und Josias Simler 1583. Bibliotheca institvta et collecta primvm a Conrado Gesnero, deinde in Epitomen redacta et novorum librorum accessione ocupletata, tertio recognita et in duplum post priores editiones aucta per Josiam Simlerum: jam vero postremo aliquot mille cum priorum tum novorum authorum opusculis ex instructissima Viennensi Austriae imperatoria bibliotheca amplificata per Joannes Jacobus Frisium. Zürich: Froschouer.

Gingerich, Owen 1988. Sacrobosco as a Textbook. Journal for the History of Astronomy (19/4): 269-273.

Gingerich, Owen 2004. The Book Nobody Read: Chasing the Revolutions of Nicolaus Copernicus. London: William Heinemann.

Gingerich, Owen 2015. Book Review: Studien zur „Sphaera“ des Johannes de Sacrobosco: Studien zur „Sphaera“ des Johannes de Sacrobosco. Hamel, Jürgen (Acta historica astronomiae, li; Akademische Verlagsanstalt, Leipzig, 2014). Journal for the History of Astronomy (46/1): 101-102.

Giuntini, Francesco, Élie Vinet, Christoph Clavius und Johannes de Sacrobosco 1629. Exposicion de la esfera de Ivan de Sacrobosco, doctor parisiense. Salamanca: I. Taberniel.

Godman, Peter 2000. The Saint as Censor. Robert Bellarmine between Inquisition and Index. Leiden; Boston: Brill.

Gorman, Michael John 1996. A Matter of Faith? Christoph Scheiner, Jesuit Censorship, and the Trial of Galileo. Perspectives on Science 3(4): 283-320.

Green, Jonathon 2005. The Encyclopedia of Censorship. New York: Facts On File.

Grendler, Paul 2007. The Conditions of Enquiry: Printing and Censorship. In: Charles Schmitt, Quentin Skinner, Eckhard Kessler und Jill Kraye (Hg.). The Cambridge History of Renaissance Philosophy. Cambridge: Cambridge University Press: 25-54.

Grendler, Paul F. 1977. The Roman Inquisition and the Venetian Press 1540-1605. Princeton, N.J.: Princeton University Press.

Hamel, Jürgen 2004. Johannes de Sacroboscos Handbuch der Astronomie. Kommentierte Bibliographie der Drucke der Sphaera, 1472 bis 1656. In: Dietmar Fürst (Hg.). Wege der Erkenntnis: Festschrift für Dieter B. Hermann zum 65. Geburtstag. Frankfurt am Main: Deutsch: $115-170$.

Hamel, Jürgen 2014. Studien zur „Sphaera“ des Johannes de Sacrobosco. Leipzig: AVA, Akademische Verlagsanstalt.

Harmening, Dieter 1979. Superstitio: Überlieferungs- und theoriegeschichtliche Untersuchungen zur kirchlich-theologischen Aberglaubensliteratur des Mittelalters. Berlin: E. Schmidt.

Hasecker, Jyri 2017. Quellen zur päpstlichen Pressekontrolle in der Neuzeit (1487-1966). Paderborn: Ferdinand Schöningh. 
Hellyer, Marcus 1996. „Because the Authority of My Superiors Commands“: Censorship, Physics and the German Jesuits. Early Science and Medicine (1/3): 319-354.

Hera y de la Varra, Pedro de la 1584. Reportorio del mundo particular de las Spheras del Cielo y Orbes elementales, y de las significaciones, y tiempos correspondientes a su luz, $y$ mouiento. Madrid: G. Druy.

Hirai, Hiro und Rienk H. Vermij (Hg.) 2017. The Marginalization of Astrology. Sonderausgabe von Early Science and Medicine. https://doi.org/10.1163/15733823-02256P01.

Hurtado Torres, Antonio 1982. La „Esphera“ de Sacrobosco enla España de los siglos XVI y XVII: Difusión bibliográfica. Cuadernos bibliográficos (44): 49-58.

Inghirami, Francesco 1844. Storia della Toscana: compilata ed in sette epoche distribuita. Bd. 13. Fiesole: Poligrafia Fiesolana.

Knobloch, Eberhard und Karin Reich 2004. Melanchthons Vorreden zu Sacroboscos „Sphaera" und zum „Computus ecclesiasticus." In: Wolfgang R. Dick und Jürgen Hamel (Hg.). Beiträge zur Astronomiegeschichte. Thun; Frankfurt am Main: Deutsch: 14-44.

Koestler, Arthur 1959. The Sleepwalkers: A History of Man's Changing Vision of the Universe. New York: Macmillan.

Labarre, Albert 1970. Editions et privilèges des héritiers d'André Wechel à Francfort et à Hanau: 1582-1627. Gutenberg-Jahrbuch (45): 209-223.

Lalla, Sebastian 2003. Über den Nutzen der Astrologie: Melanchthons Vorwort zum „Liber de sphaera.“ In: Günter Frank (Hg.). Gedenken und Rezeption: 100 Jahre Melanchthonhaus. Heidelberg: Verl. Regionalkultur: 147-160.

Lattis, James M 1989. Christopher Clavius and the "Sphere" of Sacrobosco: the Roots of Jesuit Astronomy on the Eve of the Copernican Revolution. Diss. University of Wisconsin, Madison.

Ludwig, Corinna 2010. Die Karriere eines Bestsellers. Untersuchungen zur Entstehung und Rezeption der Sphaera des Johannes de Sacrobosco. Concilium medii aevi (13): 153-185.

Lüthy, Christoph 2005. The Confessionalization of Physics: Heresies, Facts and the Travails of the Republic of Letters. In: John Hedley Brooke und Ian Maclean (Hg.). Heterodoxy in Early Modern Science and Religion. Oxford: Oxford University Press: 81-114.

Manning, Patricia 2009. Voicing Dissent in Seventeenth-century Spain: Inquisition, Social Criticism and Theology in the Case of El Criticón. Leiden; Boston: Brill.

Martins, Maria Teresa Payan 2011. O Índice Inquisitorial de 1624 à luz de novos documentos. Cultura. Revista de História e Teoria das Ideias (28): 67-87. https://doi.org/10.4000/ cultura.170.

Mascarenhas, Fernando Martins 1624. Index auctorum da[m]natce memorice, tum etiam librorum: qui vel simpliciter, vel adexpurgatione[m] vsque prohibentur, vel deniq[ue] iam expurgati permittuntur. Lissabon: P. Craesbeck.

Mauro da Firenze und Johannes de Sacrobosco 1537. Sphera volgare nouamente tradotta con molte notande additioni di geometria, cosmographia, arte nauicatoria, et stereometria, proportioni, et quantita delli elementi, di stanze, grandeze, et mouimenti di tutti li corpi celesti ... Autore M. Mauro fiorentino. Venedig: B. Zanetti.

Mauro da Firenze und Johannes de Sacrobosco 1550. Annotationi sopra la lettione della Spera de Sacro Bosco. Florenz: L. Torrentino.

Mayer, Thomas F. 2013. The Roman Inquisition: A Papal Bureaucracy and Its Laws in the Age of Galileo. Philadelphia: University of Pennsylvania Press.

Mayer, Thomas F. 2015. The Roman Inquisition: Trying Galileo. Philadelphia: University of Pennsylvania Press.

Melanchthon, Philipp 2003. Briefwechsel: kritische und kommentierte Gesamtausgabe. Bd. 5: Texte 1110-1394 (1531-1533). Herausgegeben von Walter Thüringer. Stuttgart; Bad Cannstatt: Frommann-Holzboog.

Minnich, Nelson H. 2010. The Fifth Lateran Council and Preventive Censorship of Printed Books. Annali della Scuola Normale Superiore di Pisa, Classe di Lettere e Filosofia. 2(1): 67-104.

Monfasani, John 1988. The First Call for Press Censorship: Niccolò Perotti, Giovanni Andrea Bussi, Antonio Moreto, and the Editing of Pliny's Natural History. Renaissance Quarterly 41(1): 1-31. 
Montana, Arias (Hg.) 1571. Index expurgatorius librorum qui hoc seculo prodierunt, ... juxta socri concilii Tridentini Decretum. Philippi II ... jussu et auctoritate atque Albani Ducis consilio ... in Belgia concinnatus anno 1571. Antwerpen: C. Plantinus.

Müller, Kathrin 2011. Gott ist (k)eine Sphäre. Visualisierungen des Göttlichen in geometrisch-abstrakten Diagrammen des Mittelalters. In: Reinhard Hoeps (Hg.). Handbuch der Bildtheologie: Zwischen Zeichen und Präsenz. Paderborn: F. Schöningh: 311-355.

Neuber, Wolfgang, Thomas Rahn und Claus Zittel (Hg.) 2015. The Making of Copernicus: Early Modern Transformations of the Scientist and his Science. Leiden: Brill.

Neveu, Bruno 1993. L'erreur et son juge: remarques sur les censures doctrinales à l'époque moderne. Neapel: Bibliopolis.

Nuovo, Angela 2015. The Book Trade in the Italian Renaissance. Leiden: Brill.

Omodeo, Pietro Daniel 2014. Copernicus in the Cultural Debates of the Renaissance: Reception, Legacy, Transformation. Leiden; Boston: Brill.

Oosterhoff, Richard J. 2015. A Book, a Pen, and the Sphere: Reading Sacrobosco in the Renaissance. History of Universities (28/2): 1-54.

Pagano, Sergio M. (Hg.) 2009. I documenti vaticani del processo di Galileo Galilei (1611-1741). Vatikanstadt: Archivio segreto Vaticano.

Pantin, Isabelle 1987. La lettre de Melanchthon à Simon Grynaeus: Avatars d'une défense de l'astrologie. In: École normale supérieure de jeunes filles (Hg.). Divination et controverse religieuse en France au XVIe siècle. Paris: École normale supérieure de jeunes filles: 85-101.

Paolin, Giovanna und Andrea Del Col (Hg.) 1991. L'Inquisizione romana in Italia nell'età moderna: archivi, problemi di metodo e nuove ricerche: atti del seminario internazionale, Trieste, 18-20 maggio. Rom: Ministero per i beni culturali e ambientali, Ufficio centrale per i beni archivistici.

Pardo Tomás, José 1991. Ciencia y censura: la Inquisición española y los libros científicos en los siglos XVI y XVII. Madrid: Consejo Superior de Investigaciones Científicas.

Pedersen, Olaf 1985. In Quest of Sacrobosco. Journal for the History of Astronomy (16): 175-221. https://doi.org/10.1177\%2F002182868501600302

Peixoto, Jorge 1966. Os privilégios de impressao dos livros em Portugal no século XVI. Gutenberg-Jahrbuch (41): 265-272.

Pereira da Silva, Luciano A. 1915. A Astronomia dos Lusiadas. Coimbra: Imprensa da Universidade.

Prosperi, Adriano 2003. L'Inquisizione Romana: letture e ricerche. Rom: Edizioni di Storia e Letteratura.

Prosperi, Adriano, Vincenzo Lavenia und John A. Tedeschi (Hg.) 2010. Dizionario storico dell'Inquisizione. Pisa: Edizioni della Normale.

Rebellato, Elisa 2008. La fabbrica dei divieti: gli indici dei libri proibiti da Clemente VIII a Benedetto XIV. Mailand: Sylvestre Bonnard.

Rekers, Bernard 1961. Benito arias Montana, 1527-1598: Studie over een groep Spiritualische Humanisten in Spanje en de Nederlanden, op grond van hun briefwisseling. Groningen: V.R.B. Kleine.

Reusch, Franz Heinrich (Hg.) 1886. Die Indices librorum prohibitorum des sechzehnten Jahrhunderts. Tübingen: Litterarischer Verein in Stuttgart.

Rhein, Stefan 1996. „Italia magistra orbis terrarum“. Melanchthon und der italienische Humanismus. In: Michael Beyer und Günther Wartenberg (Hg.). Humanismus und Wittenberger Reformation: Festgabe anlässlich des 500. Geburtstages des Praeceptor Germaniae Philipp Melanchthon am 16. Februar 1997. Leipzig: Evangelische Verlags-Anstalt: $367-388$.

Ricci, Saverio 2008. Inquisitori, censori, filosofi sullo scenario della Controriforma. Rom: Salerno.

Rocamora y Torrano, Ginés 1599. Sphera del universo. Madrid: Iuan de Herrera.

Rosen, Edward 1974. Rheticus as Editor of Sacrobosco. In: Robert S. Cohen, John Stachel und Marx W. Wartofsky (Hg.). Scientific, Historical and Political Essays in Honor of Dirk J. Struik. Springer, Dordrecht: 245-248.

Johannes de Sacrobosco 2008. La sfera da Gerberto al Sacrobosco. Herausgegeben von Costantino Sigismondi. Rom: Ateneo Pontificio Regina Apostolorum. 
Sander, Christoph 2017. For Christ's Sake: Pious Notions of the Human and Animal Body in Early Jesuit Philosophy and Theology. In: Lo Presti, Roberto und Buchenau, Stefanie (Hg.). Human and Animal Cognition in Early Modern Philosophy and Medicine. Pittsburgh: University of Pittsburgh Press: 55-73.

Sander, Christoph und Cristiano Casalini 2017. Benet Perera's Pious Humanism. Aristotelianism, Philology, and Education in Jesuit Colleges. An Edition of Perera's Documenta quaedam perutilia. History of Universities (30/1): 1-42.

Sandoval y Rojas, Bernardo de 1612 . Index librorum prohibitorum et expurgatorum ... Bernardi de San Doval et Roxas ... auctoritate et iussu editus. Madrid: L. Sanchez.

Schmuki, Karl 1999. Spuren jesuitischer Zensurmassnahmen im Kloster St. Gallen im 16. und 17. Jahrhundert. Schriften des Vereins für Geschichte des Bodensees und seiner Umgebung (117): 179-206.

Scribonius, Wilhelm Adolf 1581. Isagoge sphaerica methodice proposita, a Guilhelmo Adolpho Scribonio Marpurgensi. Frankfurt am Main: A. Wechel.

Selnecker, Nikolaus 1577. Delineatio Doctrinae Sphaericae. In: Nikolaus Selnecker. Propositiones Et Qvaestiones In Octo Libros Physicorvm Aristotelis: Ad Usum Studiosorum Scriptae. Libellus Physiologicus de rebus naturalibus, Elegiaco carmine scriptus, $\mathcal{E}$ in eundem Annotationes Theologicae \& Medicae [u.a.]. Omnia ante annos viginti duos praelecta iuventuti studiosae, A Nicolao Selneccero, E nunc denuo Autoris permissu edita. Leipzig: Rhamba: 630-698.

Sgarbi, Marco 2017. Renaissance Aristotelianism and the Scientific Revolution. Physis (52/1-2): 329-345.

Siorvanes, Lucas 2014. Proclus' Life, Works, and Education of the Soul. In: Stephen Gersh (Hg.). Interpreting Proclus: From Antiquity to the Renaissance. New York: Cambridge University Press: 33-56.

Skemer, Don C. 2010. Armis Gunfe: Remembering Egyptian Days. Traditio (65/1): 75-106.

Sotomayor, Antonio 1667. Index librorum prohibitorum et expurgandorum novissimus. Madrid: Di. Diaz.

Spruit, Leen 2010. Censorship and Canon: A Note on Some Medieval Works and Authors. In: Burcht Pranger, Willemien Otten, Arjo Vanderjagt und Hent de Vries (Hg.). How the West Was Won: Essays on the Literary Imagination, the Canon, and the Christian Middle Ages. Leiden: Brill: 175-194.

Squassina, Erika 2016. Authors and the System of Publishers' Privileges in Venice (1469-1545). Gutenberg-Jahrbuch (91): 42-74.

Svoljšak, Sonja und Urša Kocjan 2016. The Cat as a Printer's Trademark: The Case of the Sessa Family. The Papers of the Bibliographical Society of America (110/1): 95-116.

Thomsett, Michael C. 2010. The Inquisition: A History. Jefferson, N.C: McFarland \& Co.

Thorndike, Lynn (Hg.) 1949. The Sphere of Sacrobosco and its Commentators. Chicago: University of Chicago Press.

Valleriani, Matteo 2017. The Tracts on the Sphere: Knowledge Restructured Over a Network. In: Matteo Valleriani (Hg.). The Structures of Practical Knowledge. Cham: Springer International Publishing: 421-473.

Velcurio, Johannes 1537. In philosophiae naturalis partem omnium praestantissimam, hoc est Aristotelis de Anima libros, epitome longè doctissima. Basel: Lasius.

Vergil Pietro Bembo, Leonhard Culmann, Helius Eobanus Hessus, Philipp Melanchthon, Herennius Modestinus, Pierio Valeriano, Aelius Donatus, Federico Torresano und Paolo Manuzio 1539. P. Vergilij Maronis Opera accuratissime nunc demum recognita.: Nempe Eccloge X. argumentis, E annotationibus, ac castigationibus Helij Hessij, Philippi Melanchthonis, E Leonardi Culmani illustratce. Georgicorum lib. IIII. cum argumentis Herennij Modestini, E eorumdem annotationibus. Aneidos lib. XII. cum argumentis eiusdem Mod. annotationibus Melanchthonis, E aliorum. Culex eiusdem à Petro Bembo recognitus. Accessit in hoe singula Ioannis Pierij Valeriani lectionis uarietas asteriscis prenotata. Index insuper rerum, ac uerborum memorabilium summa diligentia, ac fide collectus, ac in studiosorum quoque gratiam nunc primum editus. Venedig: Giovanni Antonio Nicolini da Sabbio.

Vianello, Nereo 1970. Per gli „annali“ dei Sessa tipografi ed editori in Venezia nei secoli XV-XVII. Accademie e biblioteche d'Italia (38/4-5): 262-285. 
Witcombe, Christopher L. C. E. 1991. Christopher Plantin's Papal Privileges: Documents in the Vatican Archives. De Gulden Passer (69): 133-147.

Witcombe, Christopher L. C. E 2004. Copyright in the Renaissance: Prints and the Privilegio in Sixteenth-century Venice and Rome. Leiden; Boston: Brill.

Wolf, Hubert (Hg.) 2001. Inquisition, Index, Zensur: Wissenskulturen der Neuzeit im Widerstreit. Paderborn: Schöningh.

Wolters, Gereon 2015. The Silence of the Wolves, or, Why It Took the Holy Inquisition Seventy-Three Years to Ban Copernicanism. In: Wolfgang Neuber, Thomas Rahn und Claus Zittel (Hg.). The Making of Copernicus: Early Modern Transformations of the Scientist and His Science. Leiden: Brill: 42-63.

Wootton, David 2015. The Invention of Science: A New History of the Scientific Revolution. London: Allen Lane, an imprint of Penguin Books.

Zapata, Antonio 1632. Novus index librorum prohibitorum et expurgatorum. Sevilla: F. de Lyra.

Zedelmaier, Helmut 2015. Werkstätten des Wissens zwischen Renaissance und Aufklärung. Tübingen: Mohr Siebeck.

Ziegler, Jakob 1536. De solidae sphaerae constructione. In: Jakob Ziegler. Sphaerae atque astrorum coelestium ratio, natura, \& motus: ad totius mundi fabricationis cognitionem fundamenta. Basel: Valderus: 1-21.

Christoph Sander

Max-Planck-Institut für Wissenschaftsgeschichte

Boltzmannstraße 22

14195 Berlin

Deutschland

c-sander@heimat.de 